\title{
Characterization of Contrast Agent Microbubbles for Ultrasound Imaging and Therapy Research
}

\author{
Helen Mulvana, Richard J. Browning, Ying Luan, Nico de Jong, \\ Meng-Xing Tang, Robert J. Eckersley, and Eleanor Stride
}

\begin{abstract}
The high efficiency with which gas microbubbles can scatter ultrasound compared with the surrounding blood pool or tissues has led to their widespread employment as contrast agents in ultrasound imaging. In recent years, their applications have been extended to include super-resolution imaging and the stimulation of localized bio-effects for therapy. The growing exploitation of contrast agents in ultrasound and in particular these recent developments have amplified the need to characterize and fully understand microbubble behavior. The aim in doing so is to more fully exploit their utility for both diagnostic imaging and potential future therapeutic applications. This paper presents the key characteristics of microbubbles that determine their efficacy in diagnostic and therapeutic applications and the corresponding techniques for their measurement. In each case, we have presented information regarding the methods available and their respective strengths and limitations, with the aim of presenting information relevant to the selection of appropriate characterization methods. First, we examine methods for determining the physical properties of microbubble suspensions and then techniques for acoustic characterization of both suspensions and single microbubbles. The next section covers characterization of microbubbles as therapeutic agents, including as drug carriers for which detailed understanding of their surface characteristics and drug loading capacity is required. Finally, we discuss the attempts that have been made to allow comparison across the methods employed by various groups to characterize and describe their microbubble suspensions and promote wider discussion and comparison of microbubble behavior.
\end{abstract}

Index Terms-Acoustic microscopy and imaging, medical imaging, therapeutics, ultrasound contrast agents (UCAs).

\section{INTRODUCTION}

G AS microbubbles are now in widespread use as contrast agents in ultrasound imaging as a result of their exceptional echogenicity [1], [2]. Over the past two decades, their

Manuscript received April 20, 2016; accepted June 29, 2016. Date of publication October 26, 2016; date of current version January 11, 2017. The work of R. J. Browning and E. Stride was supported by the Engineering and Physical Sciences Research Council under Grant EP/I021795/1 and Grant EP/L025825/1

H. Mulvana is with the School of Engineering, University of Glasgow, Glasgow, G12 8QQ, U.K. (e-mail: helen.mulvana@glasgow.ac.uk).

R. J. Browning and E. Stride are with the Institute of Biomedical Engineering, Department of Engineering Science, University of Oxford, Old Road Campus, Oxford OX3 7DQ (e-mail: richard.browning@eng.ox.ac.uk; eleanor.stride@eng.ox.ac.uk).

Y. Luan and R. J. Eckersley are with the Biomedical Engineering Department, King's College London, London SE1 7EH, U.K. (e-mail: ying.luan@kcl.ac.uk; robert.eckersley@kcl.ac.uk).

N. de Jong is with the Biomedical Engineering, Thorax Centre, Erasmus MC, 3000 CA Rotterdam, The Netherlands (e-mail: n.dejong@erasmusmc.nl).

M.-X. Tang is with the Department of Bioengineering, Imperial College London, London, U.K. (e-mail: mengxing.tang@imperial.ac.uk).

Digital Object Identifier 10.1109/TUFFC.2016.2613991 ability to produce localized bio-effects for therapeutic applications particularly for the treatment of cancer [3], drug and gene delivery [2], opening of the blood brain barrier [4], [5], and to seed ablation of cancerous tissue during high-intensity focused ultrasound [6] has also been widely investigated. In addition, their use in super-resolution imaging techniques, which offer unprecedented detail in ultrasound imaging, is currently being explored [7], [8]. This rapid growth in exploitation has greatly increased the need for better understanding of microbubble behavior and hence for better characterization methods.

Ultrasound contrast agents (UCAs) are made up of gas microbubbles, commonly a biologically inert fluorocarbon, stabilized by a lipid, protein, or polymer shell. Several commercial agents exist, and these are supplied either as a polydisperse suspension of preformed microbubbles or lyophilized powder that is mixed with saline and agitated by the clinician prior to use. The suspensions typically contain microbubbles ranging in size from 1 to $10 \mu \mathrm{m}$ in diameter corresponding approximately to the size of a red blood cell $(\sim 8 \mu \mathrm{m})$. This is important if microbubbles are to remain within the vasculature but able to traverse capillaries. It also mitigates the risk of embolism presented by adding gas bubbles to the blood stream. In addition, particularly for the purposes of research, contrast agents can be fabricated in-house by means of sonication or microfluidics, with the specific characteristics of the resulting suspensions varying according to the fabrication technique and materials employed.

The aim of this paper is to present the key characteristics of microbubbles that determine their efficacy in diagnostic and therapeutic applications and the corresponding techniques for their measurement. In each case, we have presented information regarding the methods available and their respective strengths and limitations, with the aim of presenting information relevant to the selection of appropriate characterization methods. First, we examine methods for determining the physical properties of microbubble suspensions. Second, we examine techniques for acoustic characterization, both of microbubble suspensions and single microbubbles. Third, we review techniques appropriate to therapeutic applications, for which detailed understanding of microbubble surface characteristics and drug loading capacity is required. Finally, we discuss the methods for comparing data sets from across the literature.

\section{Counting AND SizIng}

\section{A. Introduction}

The extent to which microbubbles are able to scatter sound or generate bio-effects is a function of their oscillatory 


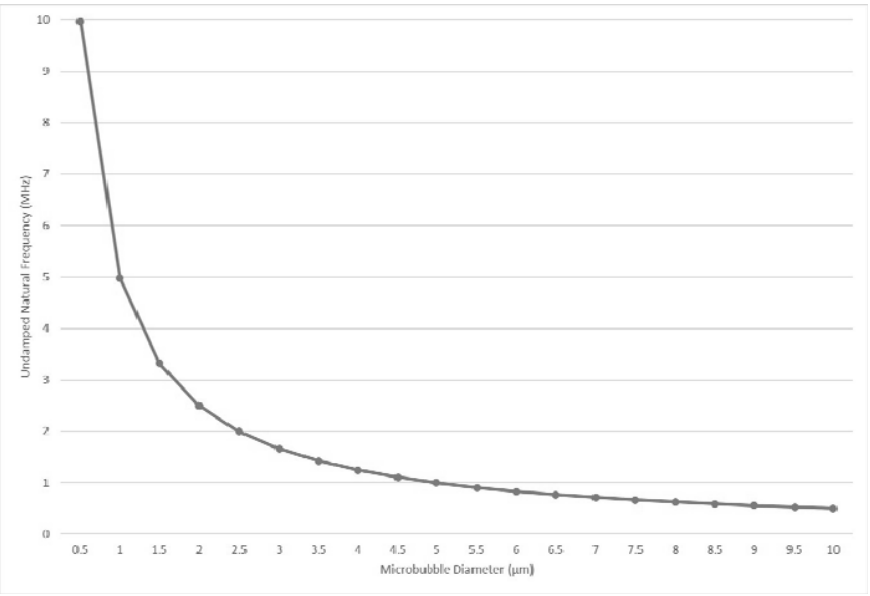

Fig. 1. Undamped linear natural frequency of an air-filled microbubble as a function of diameter calculated using Minnaert's equation [214].

behavior when subject to the alternating pressure of an ultrasound field. Upon exposure to ultrasound, the highly compressible gas core of a microbubble causes it to undergo a volumetric contraction and expansion. There is a fortunate correlation between the range of microbubble sizes that is appropriate for clinical use and that which is resonant, i.e., exhibits a maximum amplitude of expansion, at the range of frequencies commonly employed in diagnostic ultrasound imaging (1-15 MHz), as illustrated in Fig. 1. Thus, the distribution of sizes in a microbubble suspension is an important factor both in predicting their individual fundamental response [9] and in predicting the response of the population [10], [11].

Due to the range of microbubble preparations available and their variability, it is essential that researchers can easily generate detailed size and concentration information for the particular suspension they intend to use. There are several methods available for the sizing and counting of microbubbles, including electro-impedance volumetric zone sensing, typically by means of the commercial device known as a Coulter counter or multisizer (Beckman Coulter Ltd., High Wycombe, U.K.), laser diffraction (LD), commonly undertaken using a mastersizer (Malvern Instruments, Worcestershire, U.K.), and optical microscopy and single-particle optical light scattering. Each method can deliver size distribution and/or concentration measurements for suspensions of microbubbles and has particular merits and limitations. For example, commercial devices offer automation and provide swift analysis; however, the cost of investing in and operating specialist equipment may be limiting. In the case of LD, accuracy can be impaired by the reliability of the scattering model used as a basis for sizing the microbubbles due to variations in microbubble shape, the fluid they are suspended in, and accuracy with which the refractive index of the fluids involved can be estimated. Microscopy, which is more time consuming than the alternatives, offers excellent microbubble sizing accuracy. It also allows the analysis to be easily customized to deliver information about the size ranges of particular interest to ultrasound research. For example, sizing metrics arising from LD can be skewed by the high sensitivity of the technique to the sensing of nanoscale to microscale bubbles. Since bubbles of these sizes do not

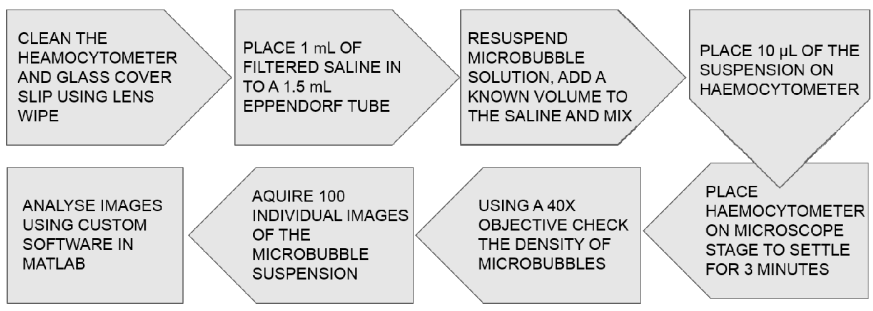

Fig. 2. Flowchart describing the main steps in optical microscopy for microbubble sizing.

contribute significantly to the echogenicity of a microbubble suspension at typical clinically applied frequencies [9], inclusion in the analysis can result in a misleading set of suspension metrics. A more detailed comparison and discussion of the merits of electro-impedance, LD, and optical microscopy can be found in [12]. This discussion has been extended by Satinover et al. [13], who compared single-particle light scattering sizing methods such as those employed in the AccuSizer (Particle Sizing Systems, Santa Barbara, CA, USA) with electrozone sensing. The authors use the generalized Lorenz-Mie scattering theory to explain the resulting differences in measurement recorded, revealing that discrepancies in sizing occur due to the nonlinearity of the relationship between the extinction cross section of light scattering from a particle and the diameter curve. They conclude that flow cytometry can provide superior sizing information compared with light obscuration methods because in the former, both the forward and side scattering measurements are used for each particle to calculate diameter.

\section{B. Optical Microscopy for Microbubble Suspension Characterization}

Optical microscopy offers a readily accessible, low-cost, and simple means of accurately sizing microbubble suspensions. The main steps in sizing a suspension in this way are illustrated in Fig. 2. The suspension is prepared, following either the manufacturer's instructions for commercial products or at the concentration it will be used at for in-house fabricated suspensions, and gently inverted to resuspend the bubbles. A known volume of the suspension is added to $1 \mathrm{~mL}$ of saline, and $10 \mu \mathrm{L}$ of this diluted suspension is added to the counting chamber of a hemocytometer using a $20-\mu \mathrm{L}$ pipette (P20). The hemocytometer is placed on a microscope stage and bubbles are allowed to rise under buoyancy to achieve a static state. Using a $40 \times$ objective, the concentration of the microbubble suspension is checked to confirm its suitability for microscopy counting. The recommendation is that there are at least 50 isolated microbubbles in the imaging frame. Should this not be the case, then the dilution step should be repeated, increasing or decreasing the volume of microbubble suspension added to the saline as appropriate. A series of individual images sufficient to include at least 5000 individual microbubbles is acquired from the hemocytometer, as representative of the suspension as a whole, and analyzed using MATLAB (The Mathworks, Inc., Natick, MA, USA) to produce sizing and concentration data as illustrated in Fig. 3. A detailed 

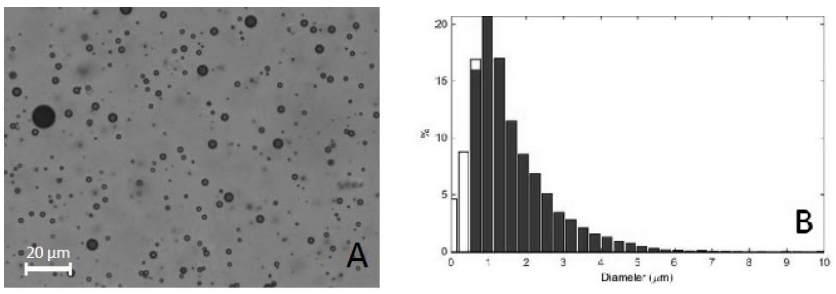

Fig. 3. Microbubble sizing using microscopy. (a) Typical bright field optical microscope image of an albumin microbubble suspension. (b) Histogram illustrating the size distribution as percentage number of the same suspension obtained using the MATLAB-based statistical analysis program described in [14].

explanation of this method of sizing microbubbles and information regarding the image analysis used can be found in [14].

\section{Optical Scattering Methods}

LD is an alternative method for sizing and counting microbubbles. In an LD analyzer (e.g., Mastersizer from Malvern Instruments, Worcestershire, U.K.), diluted particles are exposed to a laser beam and the angular light distribution and intensity scattered from the particles are measured. Particles crossing the laser beam interact with the incident beam through scattering and absorption to generate flux intensities at angles inversely proportional to the size of the individual particles. A theoretical model based on Mie or Fraunhofer scattering theories can be used to determine the particle size by fitting of the measured data. The Mie scattering model is in general more accurate for small particles, particularly those less than tens of micrometers down to tens of nanometers, but requires knowledge of the refractive indices of the particles and the medium. The refractive index of microbubbles can be determined experimentally, e.g., on a polarizing microscope using the Becke line method [15]. There are variations of the Mie model, and for microbubbles, a model that does not ignore particle absorption is recommended. Care should be taken to use appropriate concentrations in order to minimize multiple scattering at high particle densities. A study of the different sizing and counting methods [12] has shown some relatively large errors in the LD results compared with the optical microscope or the electro-impedance volumetric zone sensing. The errors are likely due to the inaccuracy in the estimation of the complex refractive indices of the microbubbles.

\section{Electro-Impedance Volumetric Zone Sensing}

Electro-impedance volumetric zone sensing "Coulter" counters and similar instruments have been widely applied to counting and sizing particles by measuring changes in the electrical impedance of a sensing zone as individual particles pass through it. In a typical setup, test particles are diluted in a conductive liquid, homogenized by stirring, and pumped through the sensing zone across which electrodes are placed. The count of changes in impedance measurements corresponds to bubble count, while the amplitude of the measurements, after calibration, corresponds to the size of the particles. A recent study has shown that a Coulter counter can generate highly reproducible results in sizing and counting microbubbles [12].

A typical Coulter counter system requires differently sized apertures to capture broad particle size distributions, as the particle size range that can be determined using a given aperture is of the order $2 \%-40 \%$ of the aperture [16], outside of which errors could be significant. While coincident passage of two or more particles through the sensing zone is a potential source of error, this can be overcome by employing a pulse discrimination system that rejects distorted pulses, which is a common feature of modern commercial systems. However, this could still potentially affect the counting results.

\section{E. Important Points to Note}

It is important to note that in order to produce data that are relevant for in vivo microbubble applications, the potential differences in environmental conditions capable of influencing microbubble stability, size, and concentration should be carefully considered. It has been shown that both the temperature and gas content of the suspending fluid can influence microbubble stability and diameter [17]-[19]. It is also widely accepted that microbubbles are particularly vulnerable to handling and rapid changes in pressure that may be encountered during transfer between solutions. Browning et al. [20] and Barrack and Stride [21] showed that the shear stresses and pressure gradients exerted by drawing suspensions through a syringe and needle can lead to microbubble destruction. In our labs, we have observed that microbubble stability can be undermined for commercial contrast agents if the septum seal is removed, exposing the suspension to the atmosphere rather than the perfluorobutane or other gas included in the head space of the vial. Each of these factors should be carefully considered both in the preparation and handling of microbubble suspensions and during examination and consideration of the results.

The relatively recent development of phase change contrast agents has offered exciting opportunities for extending the use of UCAs to the extravascular space [22]. Existing micrometer-sized bubbles are purely blood pool agents as they are too big to cross the endothelial wall (extravasation). While this is an advantage for vascular imaging, they are incapable of interrogating or delivering therapy within the interstitial space, e.g., in solid tumors. Submicrometer bubbles are difficult to make and are not efficient in providing image contrast. Phase change contrast agents are typically perflurocarbon droplets that can be prepared with nanoscale dimensions to facilitate their extravasation, after which they can be vaporized to for microbubbles using ultrasound (or other sources of energy). The characterization of such nanodroplets, however, is more challenging due to their size. While a typical optical microscope can be used to visualize some submicrometer droplets, it will not have sufficient resolution to accurately size them. Dynamic Light Scattering (DLS) is commonly used for sizing and counting such small particles. Detailed characterization of their shape and surface structure can also be achieved, for example, through the use of transmission electron microscopy (TEM). The development of higher frequency contrast imaging, requiring the use of 

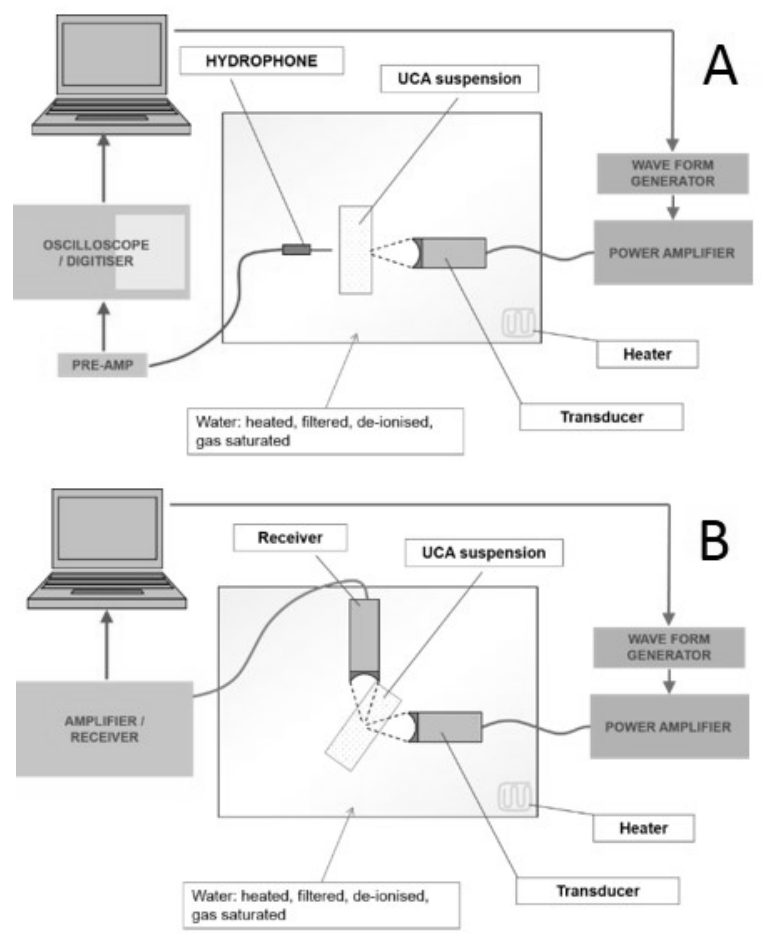

Fig. 4. Schematics showing typical arrangements for measurement of (a) acoustic attenuation and (b) acoustic scattering, where attenuation is calculated in decibels against a reference measurement taken with no microbubble suspension in the propagation path and the received signal due to scattering is calibrated against the known pressure response of the receive transducer to derive scattered pressure in kilopascals.

smaller diameter microbubbles, presents similar challenges for characterization.

\section{ACOUSTIC ChARACTERIZATION}

\section{A. Bulk Suspensions}

Bulk acoustic measurements provide information about the scattering and attenuation characteristics of a microbubble suspension and are generally undertaken to provide information that will allow acoustic performance in vivo to be anticipated. A known quantity of microbubble suspension is added to a vessel of water, itself positioned within a larger volume of water for ease of acoustic coupling. The target vessel is positioned coincident with the focus of an ultrasound transducer such that the sound can propagate through an acoustic window and through the microbubble suspension within the vessel. The suspension is gently agitated, typically using a magnetic stirrer, to ensure that the interrogation volume is regularly replenished with a fresh supply of suspended microbubbles in between measurements. Acoustic signals are then collected using either a matched transducer positioned at $90^{\circ}$ from the excitation source for acoustic scattering or a hydrophone positioned in line with the excitation source for acoustic attenuation, and acquired to a PC for postprocessing. Typical arrangements used to perform such measurements are shown in Fig. 4, and a more detailed explanation can be found in [23].

Comparable measurements can also be performed using a clinical imaging system [24]. In each case, it is important to ensure that the characteristics of the acoustic setup are removed from the data and where a clinical imaging system is used, that data can be accessed independent of proprietary postprocessing, or alternatively that steps are made to mitigate its effect, for example, by comparing data acquired under identical conditions.

\section{B. Methods of Standardizing Results for Comparison Across Research Groups}

Despite much attention having been given to the issues discussed above, ease of comparison of scattering and attenuation data across research groups can be challenging. This is due in part to the fact that no universally agreed system of comparison exists and in part to the individual environmental and setup conditions employed by different groups, as discussed in Section I. In terms of comparison, a metric that combines both scattering and attenuation measurements (linear and/or nonlinear) has been proposed in the form of an index termed the scattering to attenuation ratio (STAR) or nSTAR [25]. This index was established to describe the extent to which a contrast agent can enhance the scattered acoustic signal from a tissue perfused with contrast agent, while also presenting information about the degree to which the agent contributes to ultrasound absorption. However, the measure is equally relevant to therapeutic ultrasound in which echogenicity and treatment depth are of similar importance. Several groups have used this approach to make comparisons across microbubble formulations [26], [27]; however, it is not clear why it has not been more widely adopted.

\section{Single Microbubble Techniques}

One drawback of bulk acoustic measurements is that they do not provide any information regarding the variation in acoustic response across a microbubble population. At a given ultrasound frequency and pressure, both the amplitude and nonlinear characteristics of the oscillation of an individual microbubble are known to be highly dependent upon its size and coating properties. In order to address this, several different techniques have been developed using: 1) ultrahigh speed video microscopy, which will be addressed in Section IV; 2) acoustic scattering measurements; and 3) laser scattering measurements.

1) Acoustic Scattering:

a) Isolated capillary measurements: One of the simplest methods of measuring individual microbubble responses is to perform scattering measurements similar to those described in Section III-A, but with a highly dilute suspension of microbubbles, such that only a single bubble is present in the focal volume of the transducer at any one time. In principle, the same apparatus as described in Section III-A could be used, but in practice, some means of aligning bubbles with the transducer focus is desirable. One way to achieve this is to confine the microbubbles within an acoustically transparent tube whose diameter is large compared with the microbubble but comparable or smaller than the acoustic wavelength. For example, Casey et al. [28] utilized 200- $\mu$ m diameter cellulose dialysis tubing for this purpose. A highly dilute suspension of microbubbles is drawn into the tube by capillary action or under mild suction from a syringe pump. The capillary is suspended in a water bath at the focus of a water immersion 
microscope objective lens. Suitable positions at which isolated bubbles are located can then be identified. Ideally, the objective and ultrasound transducer should be mounted on a positioning rig such that their foci coincide. Thus, once a suitable microbubble has been identified, a scattering measurement can be made.

There are a number of drawbacks to this technique, however. First, it is relatively labor intensive and the rate at which measurements can be made is quite low. This is undesirable as it limits effective statistical analysis of microbubble population characteristics. Second, confining the microbubbles in the capillary will alter their response compared with that exhibited by an unconfined microbubble [29]. Thus, the measured responses will differ from those that might be expected in large blood vessels or predicted by theoretical models. Third, it is difficult to measure the sound field to which a microbubble will be exposed with the microscope objective in situ. Thus, either there will be some uncertainty in the exposure parameters due to scattering from the objective or the objective must be retracted immediately before the experiment at the risk of agitating the fluid and causing the bubble to move.

b) Flow focusing: An alternative means of confining microbubbles that avoids the need for a solid boundary is to create a hydrodynamically focused stream of microbubbles whose axis can be aligned with the transducer focal volume. This is achieved using a pair of coaxially aligned nozzles whose flow rates can be controlled individually, e.g., through separate syringe pumps. The nozzles are immersed in the liquid-filled chamber in which the scattering measurements are to be made. The inner nozzle carries the bubble suspension and the outer nozzle the so-called "sheath" flow that confines the bubbles to a fine stream. By adding a small amount of dye to the bubble suspension, the ultrasound transducer can be optically aligned to this stream. Roos [30] and subsequently Roy and Apfel [31] demonstrated the efficacy of this technique for the acoustic characterization of red blood cells. It was subsequently applied to microbubbles in [32] and [33].

It is still necessary to use a very dilute suspension of microbubbles to minimize the risk of multiple bubbles passing through the ultrasound focus simultaneously, but a significantly higher rate of scattering measurements can be achieved with this technique. The main drawback of this method is the lack of feedback on microbubble position and hence the precise pressure amplitude with which they are excited. Acoustic pulses are transmitted at a set repetition frequency, but there is no means of detecting where in the pressure field, a microbubble is located when a scattering measurement is recorded. Given the highly pressure-dependent nature of microbubble scattering (unlike that of solid or liquid particles), this introduces considerable uncertainty into the measurements. In addition, it is not possible to determine the initial size of each microbubble.

c) Acoustic "camera:" A more recent approach, published in [34] utilizes a similar apparatus to that described in Section III-A, but with three cofocally aligned ultrasound transducers. The first transducer is used to "drive" the microbubbles in the range $1-10 \mathrm{MHz}$, while the second and third transducers emit and receive pulses, respectively, at a much higher frequency $(30 \mathrm{MHz})$ in order to "image" the microbubbles. The imaging frequency is sufficiently above the microbubble resonance frequency for them to behave as linear scatters, and thus the recorded pressure is directly proportional to the bubble volume. The "camera" thus provides data that are more directly comparable with those obtained with ultrahigh speed imaging and can be more readily compared with theoretical predictions but with less stringent sampling frequency restrictions. In its current form, the system suffers from the same limitations as the flow focusing systems, namely, uncertainty regarding initial bubble radius and acoustic excitation pressure amplitude. Based on this acoustical measurement setup, a combined optical sizing and acoustical characterization technique was later developed [35]. A coaxial flow device was used to direct individual microbubbles to the confocal region of the ultrasound transducers and a high-resolution microscope objective. Bright field images of microbubbles flowing through the optical field of view were captured by a high-speed imaging camera synchronous with the acoustical recordings.

2) Optical Scattering: An alternative means of measuring microbubble volume is to exploit the fact that an oscillating microbubble will scatter light as well as sound. The principle is similar to that of the acoustic camera, except that the two highfrequency transducers are replaced by a light source, typically a laser, and an optical detector, such as a photomultiplier tube or photodiode. Mie scattering theory provides a direct means of determining microbubble volume from the measured optical signal.

Optical scattering measurements offer a few advantages over purely acoustic methods, albeit at the cost of additional system complexity. At low powers, a laser beam will produce negligible heating or radiation force on a microbubble. Thus, unlike a high-frequency ultrasound beam, it can be continuously transmitted throughout an experiment. This means that the laser can be used: 1) as means of determining the exact position of a microbubble and triggering the acoustic excitation and 2) as means of directly measuring microbubble volume before, during, and after that excitation.

Tu et al. [36] demonstrated this technique using a modified flow cytometer for the characterization of the commercial contrast agent SonoVue. One limitation of their experiment, however, was that the bubbles were confined by a rigid surface during the measurements and there was considerable uncertainty regarding the characterization of the sound field. More recently, Rademeyer et al. [37] utilized a similar technique, but confined the bubbles using a hydrodynamically focused flow, thereby removing the effects of a rigid boundary and enabling accurate characterization of the acoustic field. They were able to record the volumetric responses of 12000 bubbles in $30 \mathrm{~min}$ including their initial and final sizes.

All of the techniques discussed in this section are limited by the maximum signal-to-noise ratio that can be achieved in a specific setup and hence in terms of the minimum bubble size and/or amplitude of oscillation that can be detected. A further limitation is the inability to distinguish between spherical and nonspherical oscillations. This requires the use of direct imaging methods discussed in the next section. 


\section{Optical Characterization of ULtRASOUND CONTRAST AgEnTS}

Optical characterization methods rely on the acquisition of nanosecond exposure optical images of the dynamic response of microbubbles to ultrasound excitation [38], [39]. In contrast to the acoustic characterization techniques described in Section III, optical approaches are able to resolve single microbubbles, as the size of a microbubble is larger than the wavelength of light. Optical measurements based on highspeed imaging techniques can directly visualize the vibrations of individual microbubbles in response to an ultrasound field, as well as their interactions. The physical properties can be inferred by varying the applied ultrasound parameters and acquiring the radius versus time $(R-t)$ response. Moreover, various interesting phenomena (e.g., microbubble destruction and lipid shedding) specific to the applied acoustic field can be observed.

\section{A. Ultrahigh Speed Imaging Facilities}

To resolve the transient dynamics of microbubbles activated by ultrasound at megahertz frequencies, the sampling rate should exceed the central driving frequency by at least a factor of eight to ten [38]. Therefore, a frame rate of at least several millions of frames per second (fps) is required. In pioneering 1-D optical studies of microbubbles, streak imaging and strobe imaging techniques were used [40], [41]. Subsequently, 2-D ultrahigh speed photography was developed that meets both the temporal and the spatial requirements for 2-D visualization of microbubble dynamics [42].

1) Streak Imaging: Streak imaging can continuously record an event over time through a slit. For capturing microbubble dynamics, it continuously acquires a single line through the center of a bubble [40]. These 1-D images can be recorded with a nanosecond or picosecond time interval between frames. Alternatively, strobe imaging records microbubble dynamics by composing images obtained repetitively using a time-delayed single-shot method [41], [43]. However, this is not a real-time imaging method, and is restricted to repeatable events.

2) Ultrahigh Speed Photography: One approach to ultrahigh speed imaging of microbubble contrast agents is to use a shutter-controlled camera such as the Imacon 200 (DRS Hadland, Cupertino, CA, USA). In this approach, a beam splitter is used to divide an image over several recording devices, achieving a maximum recording speed of 200 million fps [44]. However, the number of maximum total recording frames available is very limited (e.g., 16 frames for Imacon). The Brandaris 128 ultrahigh speed imaging camera was introduced in 2003 by Chin et al. [45] and specifically designed with microbubble research in mind. The primary image acquired is projected onto the rotating mirror, which is mounted on a helium-driven turbine. The mirror can redirect the light beam and sweep across the lens bank and refocus the image onto 128 charge-coupled device (CCD) sensors. This optical design was also employed for the development of another high-speed rotating mirror system (UPMC Cam) [46], with further improvements in the sensitivity, spatial resolution

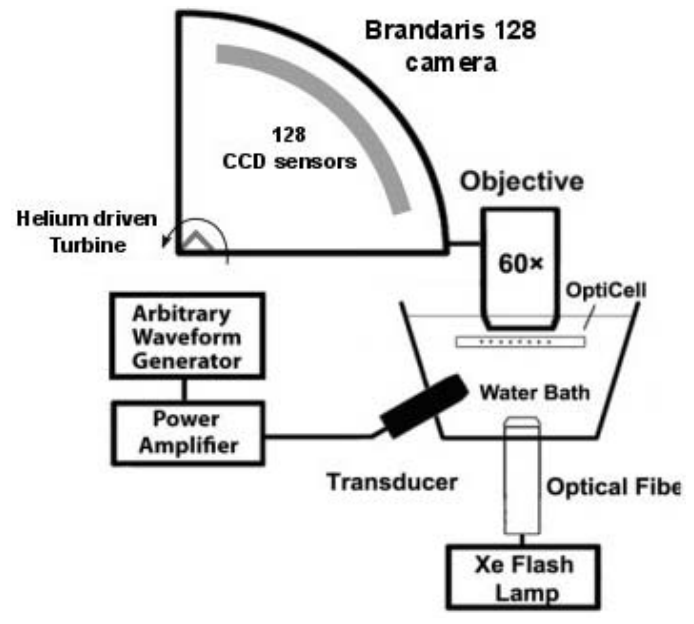

Fig. 5. Schematic of the optical setup used with the Brandaris 128 camera to optically record microbubble dynamics.

for bright field, and fluorescence imaging of microbubble dynamics. The Brandaris 128 camera exceeded earlier generations of high-speed imaging cameras in its extremely high frame rate (up to $25 \times 10^{6} \mathrm{fps}$ ) and relatively large number of recording frames (128). The camera can be coupled with various optical setups (e.g., a microscope lens) to suit the experimental conditions.

In a typical experiment, the contrast agent microbubbles are injected into a capillary tube or a chamber, which are both optically and acoustically transparent, and mounted in a water tank. The water tank is positioned below a microscope connected to the Brandaris 128 camera, and is configured with an ultrasound transducer pointing toward the optical field of view. For each individual bubble, the camera is able to record six movies of 128 frames, or 12 movies of 64 frames in a single run, which can be accomplished within a few seconds [38]. Fig. 5 shows the setup of the Brandaris 128 camera.

\section{B. Optical Characterization Techniques}

Based on the ultrahigh speed imaging camera system, many novel optical techniques have been developed to study the acoustic behavior of UCA microbubbles. Typically, the radial dynamic response is recorded at relatively low acoustic pressures (tens of kilopascals), and the radius-time curves extracted to further explore the physical properties of the agent. In the same low-pressure regime, various interesting physical phenomena have been observed, e.g., thresholding behavior [47], "compression-only" response [48], and surface mode vibrations [49]. At higher pressures (hundreds of kilopascals), the violent vibrations of microbubbles tend to disrupt the shell and are accompanied by shedding of lipid shell materials [35], [50]-[54]. Similar optical studies have also been made of other varieties of contrast agents, e.g., nanodroplets and echogenic liposomes (ELIP) [55], [56].

1) Low Acoustic Pressure Regime: The volume pulsation of a microbubble in response to an external oscillating pressure field is a function of multiple factors, e.g., the pressure amplitude, the frequency, and the physical nature of the bubble itself. As above, most clinical UCA bubbles are encapsulated in 
(a)

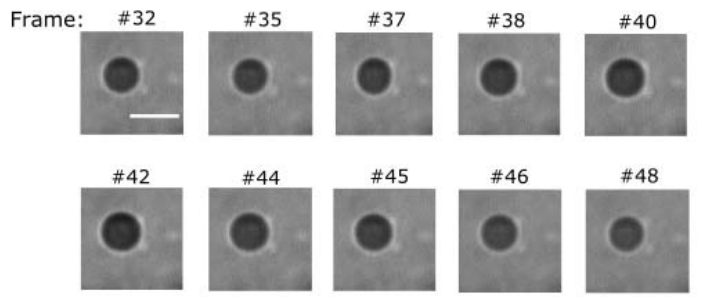

(b)

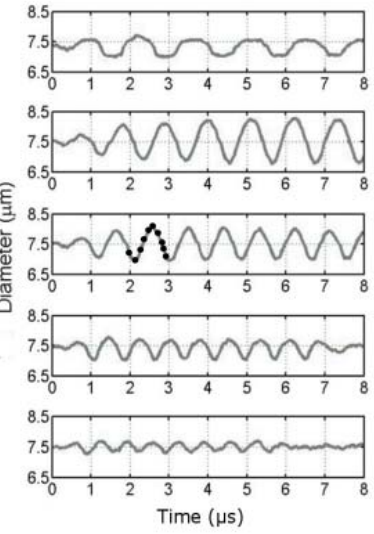

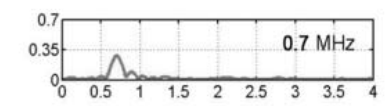
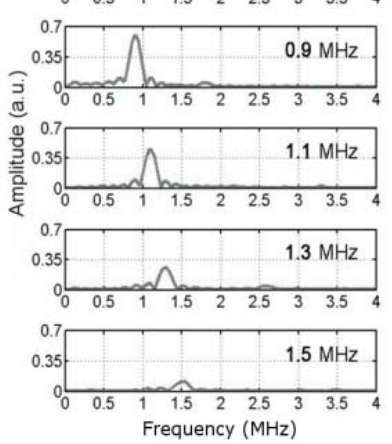

Fig. 6. (a) Example ultra-high speed image frames for a microbubble insonified by a single burst of $1.1 \mathrm{MHz}$ at the applied pressure of $50 \mathrm{kPa}$. The corresponding Diameter-time (DT) curve is shown in the third panel in (b). Black dots represent the temporal location of each image frame with respect to the DT curve. The scale bar represents $10 \mu \mathrm{m}$. (b) Radial excursions and corresponding frequency spectrums of a microbubble with a diameter of 7.5 $\mu \mathrm{m}$. The selection has been done between 0.7 and $1.5 \mathrm{MHz}$ with a step frequency of $200 \mathrm{kHz}$.

order to counter surface tension and stabilize the gas core. The presence of this outer shell can dramatically influence the microbubble acoustic behavior.

Theoretical modeling combined with optical measurement techniques has been developed to investigate the bubble dynamics at the low pressure regime, and physical properties of the bubble shell were obtained. The first model was proposed in [57], which incorporated experimentally determined elasticity and viscosity parameters into the Rayleigh-Plesset equation [58]. A more recent model was developed in [59], which considers a nonlinear effective surface tension at the bubble wall. Van der Meer et al. [60] employed a technique to characterize the shell properties, by sweeping through the driving frequency at a very low pressure $(\leq 50 \mathrm{kPa})$ in a single run of the Brandaris camera. The radius/diameter versus time curves were extracted from the videos and analyzed in the frequency domain, and resonance curves specific to single microbubbles were reconstructed. By comparing the experimental results with the simulated response for amounts of microbubbles, the elasticity and viscosity of encapsulating shell can be derived. This so-called "microbubble spectroscopy" was applied in series of studies and showed a damping effect and a shift of the resonance frequency due to the presence of the shell [61]-[64]. Fig. 6 presents an example of a recording in a "microbubble spectroscopy" experiment. Fig. 6(a) shows example ultra-high speed image frames recorded for a microbubble insonified at $1.1 \mathrm{MHz}$ under the applied acoustic pressure of $50 \mathrm{kPa}$. The corresponding Diameter-time (DT curve) is shown in the third panel in

Fig. 6(b), where the DT curves and corresponding frequency spectrums under a series of frequencies are displayed.

2) High Acoustic Pressure Regime: UCA microbubbles have also shown great potential for therapeutic use, e.g., thrombolysis [65], gene/drug delivery [66], and reversible opening of the blood brain barrier (BBB) [67]. Relatively high acoustic pressures (on the scale of several hundred kilopascals to megapascals) and long ultrasound bursts (up to several thousands of cycles) are typically used. In this pressure regime, contrast agent microbubbles can undergo dramatic changes in physical properties and vibration dynamics. Therefore, characterizing the microbubble response optically is of high clinical relevance.

a) Observations of microbubble destruction: Previous optical studies have revealed the deflation of microbubbles under relatively high acoustic pressures [51], [68]-[70], or when exposed to prolonged burst ultrasound excitations. Microbubble deflation has been ascribed to a few different physical phenomena. One is the relatively slow gas convective diffusion driven by the ultrasound exposure, which can happen within hundreds of milliseconds up to a few minutes [50]. Another process is the inertial collapse and rapid destruction of microbubbles into smaller bubbles or fragments, which is then released into the surrounding medium. The latter process can happen transiently (within a few ultrasound cycles) during violent bubble vibrations [71]. Studies based on ultrahigh speed imaging technique have shown changes in microbubble dynamic responses relative to the immediate size reduction of the microbubble [51], [70], [72].

To investigate the microbubble dynamics in response to long ultrasound pulses, a new imaging mode on the Brandaris 128 camera has been developed to extend the total time span of acquisitions [73]. The 128 CCD sensors were grouped into separate segments, with the intersegment time set to be as low as multiples of the rotation period of the turbine. Successive imaging segments were distributed over the time window of the ultrasound application. In a recent in vivo study, the segmented imaging mode was utilized to visualize the microbubble response in a chicken embryo model under a prolonged ultrasound burst lasting for over $1 \mathrm{~ms}$ [74].

b) Observations of lipid shedding behavior: There has been wide interest in gaining knowledge regarding lipid shedding behavior under the exposure to ultrasound [35], [50], [54], [75]. The loss of lipid shell material has been found to be closely related to the accompanying microbubble dissolution [51], [76]. Utilizing a high-speed imaging camera Photron (operated at 50-100 kfps), lipid shedding dynamics from fluorescently labeled microbubbles under ultrasound excitations can be clearly visualized [52]. A conventional high-speed camera can also be combined with the Brandaris 128 camera to monitor the lipid shedding behavior relative to the microbubble vibration dynamics [35]. In an experiment in which both the top view and the side view were captured, two separate physical processes were observed: the detachment of lipid particles from the microbubble shell and the subsequent transport of shed materials by the convective streaming flow field. The detachment of lipids was observed to occur within a few ultrasound cycles above a certain pressure threshold, while 


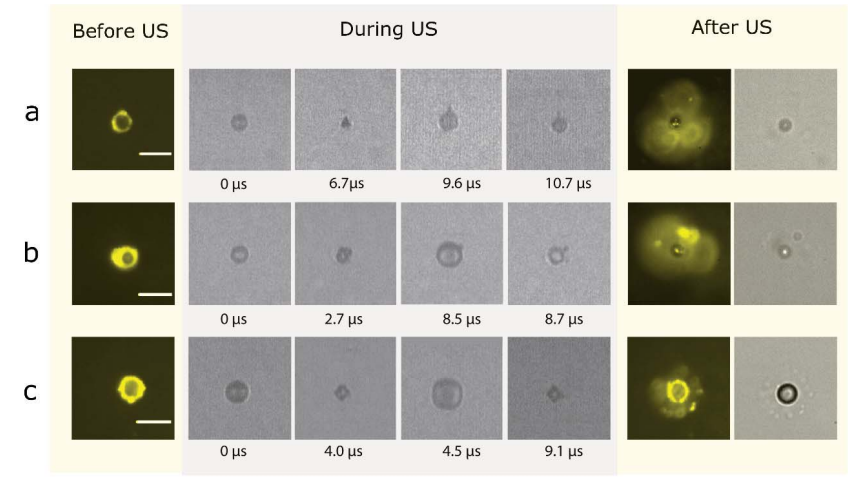

Fig. 7. (a)-(c) Examples of lipid shell material shedding shown by high-speed fluorescence imaging, recorded after the ultrasound application. Ultrahigh speed images recorded by the Brandaris 128 camera indicate the vibration dynamics and the destruction of microbubbles. A single burst of 100 cycles at above $100 \mathrm{kPa}$ [128 $\mathrm{kPa}$ for (a) and (c) and $170 \mathrm{kPa}$ for (b)] was applied. Scale bars represent $10 \mu \mathrm{m}$.

the transport trajectory was found to follow the streaming flow field and is perpendicular to the nearby cell membrane. Fig. 7 shows the shedding of lipid shell materials and the corresponding microbubble vibration dynamics and/or destruction recorded by the Brandaris 128 camera.

3) Limitations and Future Prospects: The frame rate in combination with the recording (exposure) time is in general a limiting factor for high-speed imaging facilities. For example, for the ultrahigh speed imaging camera Brandaris 128, the total exposure time of each recording is restricted to $12 \mu \mathrm{s}$ at a frame rate of $10 \mathrm{Mfps}$. Although the segmented mode was developed to counter this disadvantage, the intersegment gap inhibits a full acquisition of the bubble dynamics during the entire ultrasound exposure. Moreover, the optical setup based on a high-speed imaging camera was relatively difficult and expensive to build, comparing with other characterization techniques such as the acoustical approach. In addition, the low throughput of the high-speed imaging measurement is another disadvantage. Measuring single microbubbles requires diluting, selecting, and manipulating the bubble in a capillary or flow channel, and two people are usually required to perform a high-speed imaging experiment.

The optical system is limited by its numerical aperture and the wavelength of the light. Optical sizing of UCAs with a radius approaching the wavelength of light is difficult, as it can produce a complex bubble image strongly influenced by diffraction and scattering of the incident light. For example, the optical resolution of the Brandaris 128 camera was $0.4 \mu \mathrm{m}$ [45]. This can result in a bias of the size range of the contrast agents selected for study, especially for smaller contrast agent particles such as ELIP and Nanodroplets [77]. However, this limitation can be compensated for by fluorescence imaging, since no transmitted light is required, and bubble-background contrast can be greatly enhanced [38], [52]. Furthermore, during an optical measurement, microbubbles will reside at the top of a capillary or a chamber. Under the ultrasound excitation, the bubble does not just undergo radial excursions, but also translational motion with respect to the optical focal plane [78]. This can produce out-offocus errors depending on the amplitude of oscillation. When the amplitude is kept below $1 \mu \mathrm{m}$, the error will be on the order of $5 \%-10 \%$ [52].

\section{Surface Characteristics}

\section{A. Modeling and Mechanical Approaches}

The microbubble coating plays a key role in determining both its stability and acoustic response. Accurately measuring the properties of microbubble coatings, however, presents some significant challenges. The majority of existing theoretical models characterize the influence of the microbubble coating in terms of an effective elasticity and effective viscosity [57], [59], [79]. One way to determine these properties is to utilize one of the methods described in Section III or IV to obtain the radial response of the microbubble as a function of time and then fit the data to the theoretical model. While it can be shown that for two coating parameters, a unique solution does exist, there will inevitably be considerable uncertainty in the values obtained [80].

Alternative methods include micropipette aspiration [81] and atomic force microscopy (AFM) [82]. In the former, a small section of the microbubble coating is drawn into a glass pipette under a known pressure and the resulting deformation measured via optical microscopy. This technique, however, only provides a measure of coating elasticity under quasistatic conditions, and the relevance of this to microbubble response at ultrasonic frequencies is unknown. In AFM, a cantilever probe is either drawn across the microbubble surface or actively vibrated against it in order to produce deformation and hence determine elasticity. Again, however, the rate of deformation is slow compared with that of acoustically driven microbubble oscillations and the mode of deformation is different (bending rather than dilation).

\section{B. Fluorescence Microscopy}

In applying all of the above techniques, it is implicitly assumed that the microbubble coating can be treated as a continuous single-phase material. Several studies have now demonstrated, however, that partitioning and domain formation occur even in single-component microbubble coatings [81], [83], [84] and that these domains govern the surface microstructure and mechanical properties.

It is more appropriate therefore to consider the microbubble coating as layer of adsorbed surfactant molecules whose concentration can vary both with time (particularly if the microbubble is oscillating) and position on the bubble surface. The "elasticity" and "viscosity" of the coating are functions of this variation in molecular concentration and thus will vary with individual microbubble composition, time, temperature, and other environmental conditions. There are a number of models that have been developed on this basis [54], [59].

In order to characterize these surface properties in more detail, a variety of different quantitative fluorescence imaging techniques can be applied. In each case, a suitable fluorescent molecule needs to be incorporated into the membrane in order to probe a specific characteristic. 


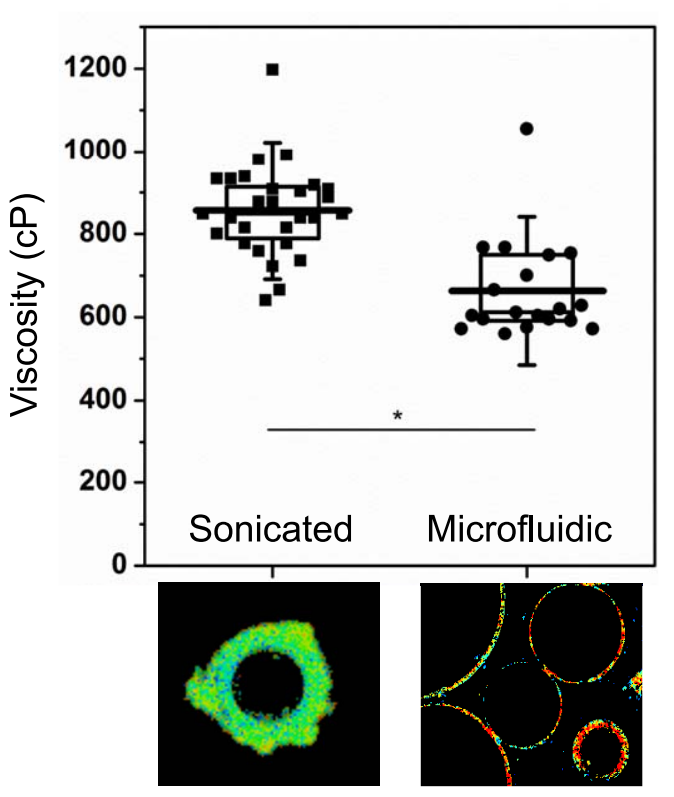

Fig. 8. Microbubble shell viscosity for microbubbles fabricated using sonication and microfluidics as measured via FLIM. Sample FLIM images are shown for both types of microbubble (adapted from [85]).

1) Fluorescence Lifetime Imaging (FLIM): FLIM can be used to investigate microbubble surface properties in combination with a specific type of fluorescent probe known as a molecular rotor. The rate of decay of the fluorescent emissions from the rotor is sensitive to its local environment. Thus, by incorporating the rotor into the microbubble coating, it can be used to map variations in molecular arrangement across the microbubble surface, as shown in Fig. 8. Examples of molecular rotors that can be used to interrogate phospholipid membranes include boron-dipyrromethene $\mathrm{C} 10$ and $\mathrm{C} 12$. These molecules preferentially locate in the hydrophobic "tail" region of the membrane and so indicate the degree of molecular interaction in this region. These interactions have been shown in previous studies to correspond to the apparent viscosity of the membrane [85].

In order to perform FLIM measurements on microbubbles, the molecular rotor needs to be combined with the microbubble coating during fabrication. This is typically done during the preparation of the lipid film [86] that is subsequently hydrated and processed to form bubbles. A fluorescence microscope with an FLIM detector is required to perform the required measurements, and the data then need to be fitted to a suitable decay model to determine the corresponding lifetimes. The Forster-Hoffman equation provides the relationship between fluorescence lifetime and viscosity.

Hosny et al. [86] reported average measurements of microbubble phospholipid coating viscosity of the same order of magnitude as values derived from high-speed camera measurements, but also showed that viscosity varied considerably both between microbubbles and over the surface of individual bubbles. One advantage of FLIM is that the timescales of intramolecular rotation (the equivalent "rate of rotation" of the rotor) correspond to those experienced by microbubbles oscillating at ultrasonic frequencies. An important point to

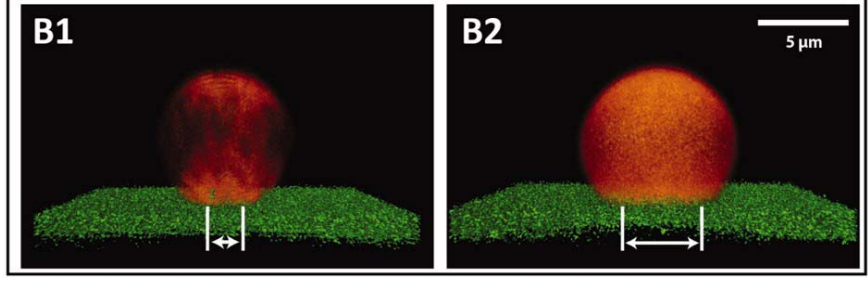

Fig. 9. Targeted DPPC microbubbles (red fluorescent) bound to streptavidincoated surface (green fluorescent; covalently linked) (reproduced from [215]).

note, however, is while the measured "viscosity" and the viscosity term appearing in theoretical microbubble models will be closely related, they may not be equal and further work is required in this area to formalize the definitions.

2) Fluorescence Recovery After Photobleaching: Kooiman et al. [87] used an alternative technique to study membrane viscosity. In their study, biotinylated phospholipid bubbles were prepared and conjugated to fluorescent streptavidin Oregon Green 488. High-resolution 3-D images (Fig. 9) were obtained using a specialized confocal laser-scanning microscope containing a matched pair of aligned opposing $100 \times$ objective lenses [88]. A high intensity laser was used to bleach the fluorophores within a specified region on the bubble, and further images were then acquired to measure the rate at which fluorescence emissions recovered from this region. This time is determined by the rate at which molecules are able to diffuse over the microbubble surface, which is itself proportional to membrane viscosity. This study also reported viscosity values consistent with those obtained from high-speed imaging although, again, the definition of viscosity requires further attention.

3) Spectral Imaging: Fluorescent probes that locate preferentially in the hydrophilic regions of surfactant membranes can also be used, e.g., Laurdan and its derivatives. The emission spectrum of Laurdan shifts as a function of the dipolar water relaxation and thus the level of hydration within its surrounding microenvironment [89], which in turn is indicative of molecular concentration or "packing." This is a potentially extremely useful measure, as both "elasticity" and "viscosity" can be directly related to it, although the specific functional relationships for microbubble coating materials have yet to be defined.

Fluorescent probes of this kind can be incorporated into microbubble coatings during preparation as above, but in this case, measurement needs to be made of the spectral content of the fluorescent emissions rather than the lifetime. This requires a microscope equipped with a detector array having the required bandwidth for a given probe. Molecular packing is defined in terms of a "generalized polarization" (GP, ranging from -1 to 1 )

$$
\mathrm{GP}=\frac{I_{440}-I_{490}}{I_{440}+I_{490}}
$$

where $I_{440}$ and $I_{490}$ correspond to the fluorescence intensity at 440- and 490-nm emission wavelengths, respectively. A high GP value corresponds to a small amount of water in the 


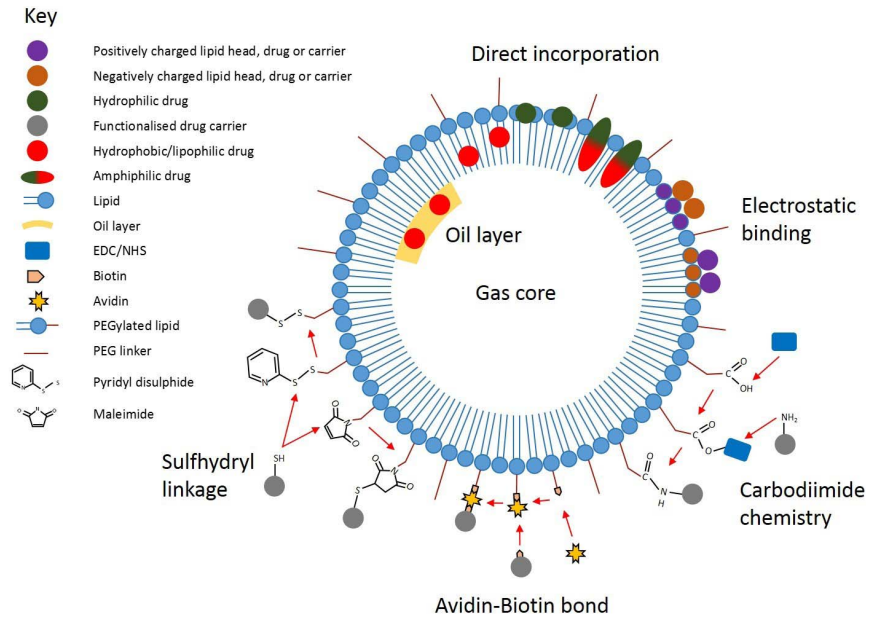

Fig. 10. Schematic of different potential loading techniques for lipid microbubbles. All techniques are applicable to protein and polymer microbubbles. Direct incorporation relies on the hydrophobic (or lipophilic), hydrophilic, or amphiphilic interactions between the drug and shell. Electrostatic binding relies on charge differences between the drug or drug carrier and shell molecules. A method not described in the text is loading the drug into an oil layer trapped beneath the shell. Approaches relying on functionalization of the shell and/or drug carrier include carbodiimide chemistry on exposed carboxylic acid $(-\mathrm{COOH})$ or amine $\left(-\mathrm{NH}_{2}\right)$ groups, tagging with avidin or biotin to form an avidin-biotin bond and using pyridyl disulphide or maleimide to link to exposed sulfhydryl (-SH) groups.

vicinity of the probe and hence to a high degree of molecular packing.

\section{Electron Microscopy}

Direct visualization of the microbubble surface at the nanoscale requires the superior spatial resolution of electron microscopy (EM). Unfortunately, the vacuum environment inside the EM system makes it extremely challenging to image microbubbles intact. Microbubbles have nevertheless been successfully examined using a variety of both scanning EM and TEM techniques.

For example, polymer-coated microbubbles have been imaged in [90] and [91]. Vlaskou et al. [92] and Hitchcock et al. [93] studied acoustically active liposomes by embedding them in gelatin blocks that were then sectioned and stained with uranyl acetate and/or lead citrate. Freeze fracture TEM has also been employed in [81] and [83] to study the surface of phospholipid coated microbubbles. Owen and Stride [84] recently proposed a low-cost method for examining microbubbles intact under TEM, using the uranyl acetate stain itself to stabilize the bubbles. This enabled visualization of nanoparticles embedded within the microbubble coating.

\section{DRUG LOADING}

\section{A. Characterization of Microbubble Chemistry}

Drug loading of microbubbles can be achieved in multiple ways, as illustrated in Fig. 10, and accurate measurement of the amount loaded is vital in order to determine the availability for delivery to a target area. This section will review the main methods for determining the drug loading capacity of a microbubble and quantity of drug actually loaded.

\section{B. Quantification of Loading Capacity}

1) Direct Incorporation: The simplest method for loading is direct incorporation of the drug into the shell of the microbubble. Lipid and protein microbubbles have an amphiphilic structure, which allows loading of amphiphilic, hydrophilic [94], or hydrophobic drugs [95]-[97]. Albumin in particular has excellent inherent capability as a drug carrier [98], [100]. This method is simple to employ and drugs are rapidly released upon ultrasonic activation, but the drug may influence shell stability, and microbubble behavior and release may occur in areas where microbubbles are trapped, for example, the lungs [101], spleen [102], [103], or liver [104], [105]. The drug loading capacity of microbubbles could be estimated using known parameters of drug loading area, hydrophobicity, and drug solubility; however, quantification of the loaded drug, by the methods described later, is simpler and more accurate.

2) Electrostatic Incorporation: Direct incorporation of hydrophilic drugs or drug carriers onto the surface of microbubbles can also be achieved by electrostatic interaction [94], [106]-[108]. Designing a microbubble with the opposite charge of the drug or drug carrier-which may itself be modified to achieve the desired charge-will create an electrostatic force that binds the two components together.

In microbubble chemistry, hydroxyl, phosphate, and carboxylic acid groups will confer a negative charge. Positive charges are commonly provided by protonated nitrogen groups, e.g., amine, trimethyl amine, etc. The presence of these groups can be confirmed by many standard chemical tests; however, the charge conferred is of primary interest. Zeta potential measurements, while not specifically the charge present at the surface of the molecule, provide a useful means for determining the charge on a molecule. However, there are some challenges associated with measuring microbubbles in commercial machines as discussed below. Due to their semirigid cross-linked nature, shell remnants of destroyed protein microbubbles should yield a relatively good estimation of zeta potential, but this is not necessarily the case with all materials. Consideration must also be given to both the buffering solution and effect in plasma, as the charge of a molecule will change with $\mathrm{pH}$ and ionic strength. This may also be of use for site-specific release in tumors where the high metabolic rate in tumor cells and excretion of $\mathrm{H}^{+}$ions result in acidification of the tumor environment [110]-[112].

a) Zeta potential measurement: Zeta potential of particles can be obtained through laser Doppler electrophoresis (LDE), which measures MB electrophoretic mobility in an electric field by light scattering and calculating its zeta potential. However, the main limitation of this method is that the buoyancy force acting on the bubbles significantly affects their measurement as commercial systems typically have a vertical. An alternative approach reported in [109] uses a simple microfluidic device for obtaining the zeta potential of three types of commercial microbubbles. The microbubbles are suspended in a horizontal microchannel under an electrical 


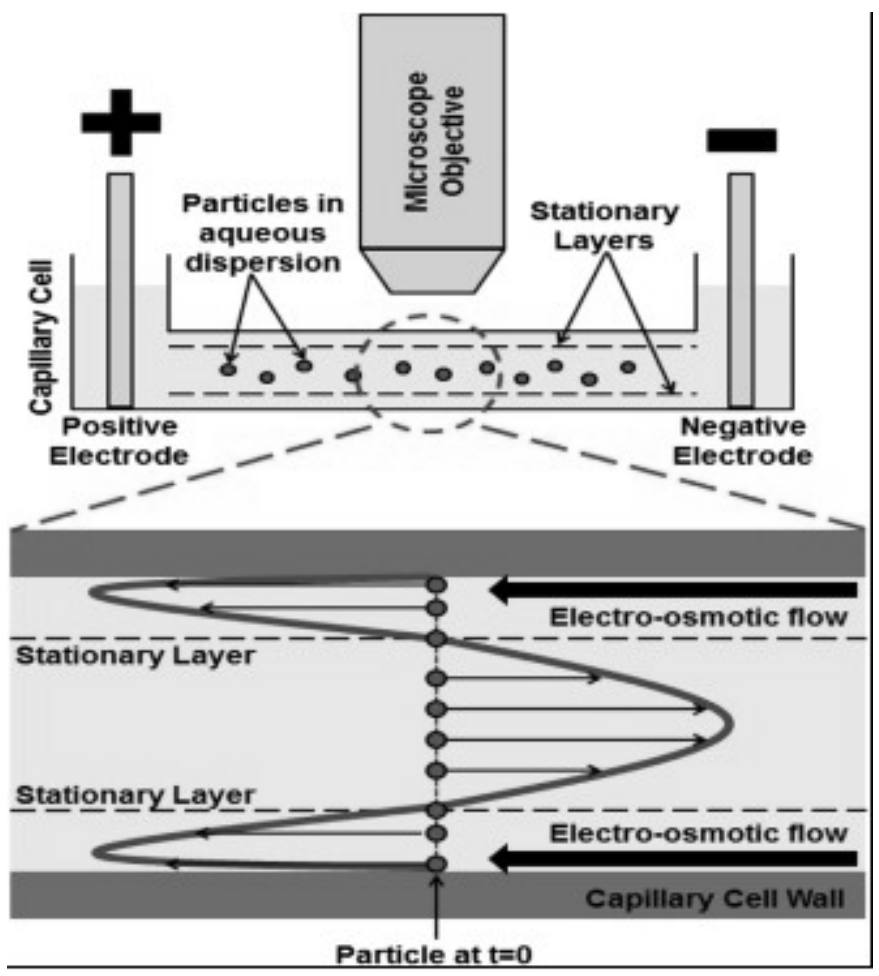

Fig. 11. Experimental setup for measuring the zeta potential of microbubbles as used in [109].

field and optically tracked as illustrated in Fig. 11. The velocity of the bubbles is then used to calculate their zeta potentials. By comparing LDE and the homemade device, a tenfold improvement in measurement variation was achieved. It should be noted that coating of the channel is needed to avoid MBs sticking to the wall as well as reducing electro-osmotic flow on the walls.

3) Conjugation of Drug Carriers: In many cases, permanent attachment to a microbubble shell may be necessary. In these instances, the drug is typically loaded into a secondary drug carrier, i.e., polymeric nanoparticles or liposomes, and attached to the microbubble via electrostatic or chemical conjugation. This improves colocalization of the drug with the microbubble at the site of activation and can help reduce systemic toxicity by drug encapsulation. In addition, conjugation of drug carriers to the shell can potentially increase the loading capacity per microbubble in comparison with incorporation into the shell. Kheirolomoom et al. [113] approximately calculated up to 10000 liposomes of $100 \mathrm{~nm}$ were loaded via avidin-biotin linkages on to single microbubbles with mean diameters of $1.7 \mu \mathrm{m}$. Compared with microbubbles only, the attachment of these liposomes can give a nearly 35-fold increase in the potential surface area alone [114].

Many conjugation techniques are available and many protocols exist for their use, in both the literature and from commercial suppliers. Direct conjugation of a carrier to a shell is possible via functional ligands; however, linker molecules are typically employed to separate the drug carrier from the shell. A popular linker is polyethylene glycol (PEG), due to its low toxicity, low cost, the wide availability of different forms and conjugates, and the ease of manipulation. PEG chains with two differing functional groups - a heterobifunctional ligand-can allow for very precise loading of desired carriers with low interference.

For protein microbubbles, the PEG linker should be conjugated onto the protein shell postproduction. Preproduction conjugation may interfere with functional groups required for stable shell synthesis, although, with controlled reactions, incomplete usage of functional groups could be attempted. For lipid microbubbles, conjugation can be performed preproduction to reduce the number of handling steps of fragile microbubbles. Alternatively, PEG-lipid conjugates with functional groups are already widely available and can be incorporated directly during microbubble production. Unreacted PEG molecules can be quantified by refractive index detection after separation from microbubbles by washing and size exclusion chromatography [115]. In addition, quantification of the relative and absolute amounts of specific lipids incorporated into the shell could be performed using gas chromatography (GC) [116], [117], Fourier transform infrared (FTIR) spectroscopy [118], or high performance liquid chromatography (HPLC) [119], [120] on washed microbubbles. HPLC combined with an evaporative light scattering (ELS) detector has been used to determine the amounts of lipids in commercial and research microbubble formulations [119], but has yet to find widespread use in the field potentially due to the low amounts of lipids used and complexity of establishing the technique [121]. Alternatively, characterization of the available functional groups on microbubbles can be attempted. A few examples of the most common functional groups will be covered here.

4) Avidin-Biotin: Avidin-biotin is one of the strongest noncovalent bonds commonly available to researchers. It is simple to use, and many PEG linkers or PEG-lipid conjugates with either biotin or avidin ligands are available. It is highly tolerant to a wide range of buffering conditions [122], [123] and is common in the preparation of novel microbubble delivery or imaging agents [113], [124]-[126]. However, the bond is practically irreversible, requires additional washing and handling steps, and avidin is potentially immunogenic, although recent research has shown that the last point may not be of as great a concern as previously thought [127]-[129].

Qualitative determination of incorporation into a microbubble shell can be achieved by widely available fluorescent biotin or avidin. Quantitatively, colorimetric and fluorimetric tests using 2-(4'-hydroxyazobenzene) benzoic acid (HABA) are available to rapidly determine biotin levels in a sample [130], [131]. This has been performed for biotinylated albumin to determine the binding efficiency of the conjugation of biotin to albumin subsequently used to create microbubbles [132] and on antibodies to be conjugated to microbubbles via avidin-biotin [133]. However, considering the low proportion of biotinylated lipids used in formulations, typically ranging from $5 \%$ to $10 \%$ molar ratio, microbubbles have not been directly investigated in this manner yet as even fluorimetric HABA assays may struggle to determine biotin quantities. 
5) Carbodiimide Chemistry: In carbodiimide chemistry, a carboxylic acid group is reacted with 1-ethyl-3-(3dimethylaminopropyl) carbodiimide (EDC), typically in the presence of $\mathrm{N}$-hydroxysuccinimide (NHS) or $\mathrm{N}$-hydroxysulfosuccinimide (sulfo-NHS) to improve yield, forming a ligand capable of forming a covalent bond with amine groups. Carbodiimide chemistry is popular as a protein linker ligand, as amine and carboxylic acid groups are common in protein residues. It is also popular for adding other functional groups to a molecule, e.g., sulfhydryls, avidin, biotin, etc. Typically, linkers use NHS due to its increased stability; however, the reaction can be performed on carboxylic acid or amine groups already present on the molecules without further manipulation.

A lipid or protein microbubble shell with available carboxylic acid groups can be modified by EDC/NHS to bind amine functionalized linkers or drug carriers [134], or vice versa [135]. Lipid-PEG-NHS conjugates are available for direct incorporation during lipid microbubble manufacture. The carbon-nitrogen bond formed is "irreversible," and this may be a disadvantage for microbubble release. In addition, the reaction can be challenging to perform and optimize as reagents are very sensitive to $\mathrm{pH}$, temperature, and time.

Quantifying EDC or NHS availability in the microbubble shell can be a challenge as stability is dependent upon $\mathrm{pH}$ and both will cleave over time. Measuring the concentration of unreacted groups may be easier. EDC, and its hydrolyzed decay products, can be detected by mass spectroscopy [136] and colorimetric methods [137], [138]. NHS and sulfoNHS have strong absorbance at $260 \mathrm{~nm}$ under basic conditions [139], and their unreacted conjugates can be isolated and detected by fluorescence assay [140] or HPLC-UV using a reversed phase column. Alternatively, during a reaction, released NHS and sulfo-NHS can be quantified by HPLC-UV using a hydrophilic interaction chromatography column [141]. Indirectly, a before and after assay of available amine, e.g., 2, 4, 6-trinitrobenzene sulfonic acid (TNBS) assay [142], [143], and carboxylic acid groups, e.g., 5-bromomethyl fluorescein assay [144], [145], can determine site usage. Finally, an amine displaying a fluorescent compound can be targeted to the activated EDC/NHS groups to determine availability on the shell.

6) Sulfhydryl Linkages: On proteins, sulfhydryl groups are less common than amine groups, increasing reaction selectivity and potentially reducing interference of protein microbubble formation. There are also many methods for adding sulfhydryls to target drug carriers via other functional groups, for example, Traut's reagent [146] or $N$-succinimidyl S-acetylthioacetate variants that target amine groups. The availability of these sulfhydryl groups can be quantified by several methods [147]-[149], and commercial assay kits are available [150], [151]. Several functional groups are capable of binding to sulfhydryl groups; however, for microbubbles, the authors could only find literature for the use of maleimide [124], [152]-[154] and pyridyl disulfide [155]-[157].

Maleimide reagents are more stable than EDC/NHS and form permanent thioether linkages. The presence of maleimide groups can be detected using commercially available fluorimetric assay kits [158] or spectrophotometric approaches [159]. The maleimide group remains in the compound postreaction as a small linker.

Pyridyl disulfide forms a direct disulfide bond with sulfhydryl groups with no linker length and release of the pyridine-2-thione, which can be monitored by absorbance at $340 \mathrm{~nm}$. This release can also be used to quantify the availability of functional groups as the pyridyl disulfide bond cleaves under reducing conditions, for example, in the presence of dithiothreitol, glutathione, or tris(2-carboxyethyl)phosphine hydrochloride. Although pyridine-2-thione has a relatively low molar extinction coefficient that may hinder monitoring of the reaction at low concentrations [160], Yoon et al. [157] were able to utilize UV/VIS detection at 270 and $340 \mathrm{~nm}$ to monitor the conjugation of pyridyl disulfide microbubbles to thiolated liposomes. The bond formed with the drug carrier is similarly not stable, which may allow drug release in the reducing environment of tumors [161].

\section{Drug Loading Quantification}

Microbubbles have been developed beyond their original diagnostic purpose into effective targeted drug delivery agents. As with all drug therapies, knowledge of the dose delivered is fundamental to ensuring a consistent therapeutic effect without adverse events or severe toxicity. Quantification of drug loading onto microbubbles is therefore vital, and the following section will cover four broad drug types currently in use with microbubbles. It should be highlighted, however, that the characterization technique required will be entirely dependent upon the drug, availability of equipment, cost, and convenience. Furthermore, few drugs considered for targeted microbubble and ultrasound delivery are novel. Literature will likely be available describing standard methods of quantification for the drug and should form the principle basis for any choice in protocol.

Ideally, quantification should be carried out after loading, either as a subtraction of unbound drug from total used or as the amount of drug still in the sample. It should be noted though that techniques to quantify the total loaded drug may not represent actual release when used, some drugs may become irreversibly bound to drug carrier or microbubble and not be available. Loading can be measured on either intact or destroyed microbubbles; however, the buoyant and light scattering characteristics of intact microbubbles can interfere with some measurement techniques. Negative pressure, sonication, and heat are effective methods for microbubble destruction. In all cases, effective washing will be required to remove contaminants. This can be challenging with microbubbles due to their sensitivity to pressure, shear stress, and handling. Centrifugation is the most common method for washing microbubbles, but will inevitably cause some concentration loss and change in size distribution [162]. For sufficiently stable microbubble complexes, buoyancy over time may allow purification [163]-[165].

If the drug is difficult to detect, or difficult to separate from the influence of the carrier or microbubble, quantification of the drug carrier could be attempted instead. Fluorophores, either attached to the carrier or carried with the drug, allow 
easy quantification of loading onto microbubbles by spectrophotometry. In addition, fluorescent drug carriers can act as convenient markers, for example, of delivered "dose," delivery distances from blood vessels, distribution throughout target tissue, off-target dose, and method of excretion. Radiolabeled drug carriers may also fulfil this role and can be monitored noninvasively by positron emission tomography (PET) [166] or single photon emission computed tomography (SPECT), although this approach has increased risk, complexity, and cost. Drug carriers can also be tagged with magnetic resonance imaging (MRI)-sensitive agents, but the equipment required for quantification of the carrier and monitoring of delivery may be prohibitive.

\section{1) Cargo Types:}

a) Genetic material: Genetic material is defined here as any form of long or short chain of nucleotides, single or double stranded, including, but not limited to, deoxyribonucleic acid (DNA) [167], ribonucleic acid (RNA), messenger RNA (mRNA), small interfering RNA (siRNA) [94], [168], short hairpin RNA (shRNA), and potentially, some forms of simple virus. The choice of genetic material will depend upon application. From a loading perspective, the most important feature shared between different types of genetic materials is the presence of a negatively charged sugar-phosphate backbone. Cationic drug carriers, such as polyethyleneimine (PEI) [169], will load genetic material by electrostatic attraction. This loading can also protect from nuclease degradation in vivo [170], [171]. In addition, the neutralization of the negative can condense the genetic material to improve loading and transmembrane passage [172].

In genetic material, the phosphate $(\mathrm{P})$ groups confer a strong negative charge. Cationic drug carriers are normally formulated with protonated nitrogen groups $(\mathrm{N})$, e.g., amine, imizadole, trimethyl ammonium, etc. The N/P molar ratio is commonly used to optimize electrostatic loading for genetic material [173], [174]. As the backbone is a repeating structure, $\mathrm{P}$ can be calculated from the length of the genetic material, which is either known or measurable by standard electrophoretic techniques. For N, values for standard cationic drug carriers, e.g., PEI, are available in the literature. Cationic lipid components could be estimated from the initial ratios of chemical components; however, accurate estimates can only be achieved via analysis of the lipid constituents post production, as mentioned earlier. Alternatively, and specifically for protein microbubbles, there are many tests available, which can detect protonated nitrogen groups to estimate $\mathrm{N}$, for example, the TNBS assay for primary amines [142]-[144].

Loading can be qualitatively demonstrated by measuring changes in zeta potential charge as previously mentioned, or by the use of nucleic acid specific dyes or stains, for example, ethidium bromide, SYBR derivatives, 4',6diamidino-2-phenylindole, YOYO-1, Hoescht, and propidium iodide [170], [175].

Many different options are available for quantitative measurement of genetic material. For lipid microbubbles without aromatic components, the simplest technique available is UV spectroscopy after microbubble destruction [170], [171]. However, the correct calibration curve must be chosen, or the genetic material purified, as different forms of genetic material, its structure, contaminants, and buffer will affect the absorption profile. Sensitivity of spectrophotometric methods can be increased and contamination issues are avoided by the use of the aforementioned dyes, although they do increase cost and are usually hazardous. These stains may be essential for protein microbubbles without purification as some amino acid residues absorb in the UV range [176]. Alternatively, genetic material can be radiolabeled to allow sensitive quantification on intact microbubbles without issues of interference or altering loading properties [177]-[179]. However, this does further increase cost, complexity, and potential risks.

An alternative method for both quantification and checking for degradation of the genetic material is gel electrophoresis. Separation of genetic material by electrophoresis can highlight structural abnormalities by differences in migration speed against controls [180], [181]. This method can also be used to quantify genetic material if run against known concentrations [182].

For all the techniques mentioned above, it should be noted that microbubbles may leave behind charged shell remnants after destruction, which could interfere with subsequent analyses. Removal of electrostatically bound material may be required, for instance, dissolution in $4 \mathrm{M}$ guanidine hydrochloride [183]. Alternatively, standalone kits and techniques are available to isolate genetic material from proteins. Increasing the alkalinity of the sample could be attempted to the deprotonate the nitrogen groups, but this should be done at low temperatures as a high $\mathrm{pH}$ will result in degradation of most genetic material, particularly RNA [184].

(i) Chemotherapy drugs: An active area of microbubble research is loading of established chemotherapy drugs, such as doxorubicin [108], [168], [185]-[189], 5-fluorouracil [190], and paclitaxel [97], [191], [192]. As chemotherapy drugs have high systemic toxicity, the ability to direct delivery to tumor tissue is an attractive therapy. Quantification of established chemotherapy drugs will be well described in the literature due to their clinical use, and the following will be general guidance on techniques.

For some chemotherapy drugs, direct quantification may be possible by simple fluorescence spectrophotometry, for example, rose Bengal [193], [194], protoporphyrin IX [195], [196], topotecan [197], [198], doxorubicin [199], and irinotecan [200]. This also allows qualitative demonstration of loading and delivery by fluorescent microscopy and histology techniques.

For nonfluorescent drugs, alternative techniques must be considered. Release and separation of the drug from the microbubble or drug carrier can be followed by quantification. Drug carriers, such as liposomes, can be disrupted by heating to the lipid phase transition temperature, whereas polymeric nanoparticles like PLGA can be dissolved in solvents or broken down with sodium hydroxide.

For most drugs, literature will be available for a variety of techniques and separation criteria. Automated methods for separation, common in most laboratories, are HPLC, GC, or asymmetrical flow field flow fractionation (AF4). Detection is usually performed by UV 
absorbance, although many different detectors are available, including mass spectrometry, ELS, and multilight scattering detector. For example, HPLC-UV methods have been described for cisplatin [201], mitomycin C [202], and 5-fluorouracil [203]. In addition, certain drugs, such as photodynamic or sonodynamic therapy drugs, generate detectable free radicals and reactive oxygen species upon activation [204], [205], which could be used for drug quantification.

(ii) Proteins: Loaded proteins can include hormones [126] or growth factors [206], [207]. Potentially, the proteins could also act as a drug carrier, for instance, albumin nanoparticles [98], [99], [115], [208]. Protein detection can be performed by UV spectroscopy at $280 \mathrm{~nm}$, but the sensitivity is low, extinction coefficient is dependent upon sequence, and many contaminants absorb in the same range. Instead, many colorimetric or fluorescent assays are available for protein quantification, e.g., the Lowry method, bicinchoninic acid assay, and Quant-iT (see [209] for a review). Fluorescence methods also allow qualitative assessment of loading on to intact microbubbles. Protein samples can also be examined on HPLC-UV machines by several methods [210], [211]. Finally, if the protein has some intrinsic enzymatic activity, an assay could be used to quantify both loading and integrity of the sample.

(iii) Cells: Stem cell delivery using acoustic radiation force and microbubbles is another area currently under research [189], [212], [213]. In these cases, loading is "reversed," with one cell potentially loading multiple microbubbles via antibodies. Optical microscopy is usually sufficient to determine microbubble loading, although fluorescent staining of the bubble with lipophilic dyes or fluorophore conjugation would allow automated techniques such as fluorescence-assisted cell sorting (FACS) to be used. FACS would also allows sorting of cells with desired levels of microbubble loading and a rough estimate of cell number can be determined from absorbance at $600 \mathrm{~nm}$, although this technique should be performed without microbubbles to avoid light scattering effects.

\section{CONCLUSION}

This paper presents the means by which different research groups have sought to characterize and compare microbubbles for diagnostic and therapeutic applications. It is important to note that for each of the characteristics discussed, comparison across research labs or indeed between different investigations within the same lab is possible only when careful consideration of the environmental and setup conditions that may influence microbubble behavior has been made. In addition, where results are to be used to gain insight to behavior in vivo, careful control of temperature, gas saturation, and preparation and handling methods used are essential to give reliable results. It is clear that while the case for developing a standardized method of comparison for microbubble contrast agents is strong, several challenges remain to support translation of new formulations for therapy and emerging quantitative imaging techniques from research to practice.
The characterization of polydisperse microbubble suspensions by mean diameter does not capture the complexities of size distribution or echogenic behavior. There is certainly a case for including alongside this information details of the gas volume distribution and description of the suspensions polydispersity characteristics. There is no consensus on a reliable and useful means of comparing microbubble acoustic behavior. The development of the STAR method has contributed to this discussion by providing a useful and easily understood means of comparison, but has not seen wide adoption. This may be in part because commercial agents, which are considered to be well characterized, remain dominant for in vivo investigations. It is also possible that the STAR/nSTAR value could be similar for a contrast agent suspension that does not attenuate or scatter effectively to one that both attenuates and scatters effectively. However, our growing understanding of how environmental factors influence microbubble behavior, the continued development of new, and particularly in-house fabricated microbubbles which are particular to each research group, plus our demands for improved insight into microbubble biophysics to understand therapeutic applications and extend their utility ensures that the need to accurately characterize microbubbles will remain important.

\section{REFERENCES}

[1] J. R. Lindner, "Microbubbles in medical imaging: Current applications and future directions," Nature Rev. Drug Discovery, vol. 3, pp. 527-533, Jun. 2004.

[2] S. Hernot and A. L. Klibanov, "Microbubbles in ultrasound-triggered drug and gene delivery," Adv. Drug Del. Rev., vol. 60, no. 10, pp. 1153-1166, 2008.

[3] S. Ibsen, C. E. Schutt, and S. Esener, "Microbubble-mediated ultrasound therapy: A review of its potential in cancer treatment," Drug Design, Develop. Therapy, vol. 7, pp. 375-388, May 2013.

[4] E. E. Konofagou, "Optimization of the ultrasound-induced blood-brain barrier opening," Theranostics, vol. 2, no. 12, pp. 1223-1237, 2012.

[5] H.-L. Liu, C.-H. Fan, C.-Y. Ting, and C.-K. Yeh, "Combining microbubbles and ultrasound for drug delivery to brain tumors: Current progress and overview," Theranostics, vol. 4, no. 4, pp. 432-444, 2014.

[6] G. Ghoshal and M. L. Oelze, "Enhancing cell kill in vitro from hyperthermia through pre-sensitizing with ultrasound-stimulated microbubbles," J. Acoust. Soc. Amer., vol. 138, no. 6, pp. EL493-EL497, 2015.

[7] C. Errico et al., "Ultrafast ultrasound localization microscopy for deep super-resolution vascular imaging," Nature, vol. 527, pp. 499-502, Nov. 2015.

[8] K. Christensen-Jeffries, R. J. Browning, M.-X. Tang, C. Dunsby, and R. J. Eckersley, "In vivo acoustic super-resolution and superresolved velocity mapping using microbubbles," IEEE Trans. Med. Imag., vol. 34, no. 2, pp. 433-440, Feb. 2015.

[9] J.-M. Gorce, M. Arditi, and M. Schneider, "Influence of bubble size distribution on the echogenicity of ultrasound contrast agents: A study of SonoVue," Invest. Radiol., vol. 35, no. 11, pp. 661-671, 2000.

[10] K. Cheung et al., "In vitro characterization of the subharmonic ultrasound signal from definity microbubbles at high frequencies," Phys. Med. Biol., vol. 53, no. 5, p. 1209, 2008.

[11] B. D. Lindsey, J. D. Rojas, and P. A. Dayton, "On the relationship between microbubble fragmentation, deflation and broadband superharmonic signal production," Ultrasound Med. Biol., vol. 41, no. 6 , pp. 1711-1725, 2015.

[12] C. A. Sennoga et al., "Evaluation of methods for sizing and counting of ultrasound contrast agents," Ultrasound Med. Biol., vol. 38, no. 5, pp. 834-845, 2012.

[13] S. J. Satinover, J. D. Dove, and M. A. Borden, "Single-particle optical sizing of microbubbles," Ultrasound Med. Biol., vol. 40, pp. 138-147, Jan. 2014.

[14] C. A. Sennoga et al., "On sizing and counting of microbubbles using optical microscopy," Ultrasound Med. Biol., vol. 36, no. 12, pp. 2093-2096, 2010. 
[15] P. F. Kerr, Optical Mineralogy, 4th ed. London, U.K.: McGraw-Hill, 1977.

[16] T. Allen, Particle Size Measurement, 4th ed. London, U.K.: Chapman \& Hall, 1990.

[17] H. Mulvana, E. Stride, J. V. Hajnal, and R. J. Eckersley, "Temperature dependent behavior of ultrasound contrast agents," Ultrasound Med. Biol., vol. 36, no. 6, pp. 925-934, 2010.

[18] H. Mulvana, E. Stride, M.-X. Tang, J. V. Hajnal, and R. J. Eckersley, "The influence of gas saturation on microbubble stability," Ultrasound Med. Biol., vol. 38, no. 6, pp. 1097-1100, 2012

[19] V. Sboros, C. M. Moran, T. Anderson, and W. N. McDicken, "An in vitro comparison of ultrasonic contrast agents in solutions with varying air levels," Ultrasound Med. Biol., vol. 26, no. 5, pp. 807-818, 2000

[20] R. J. Browning, H. Mulvana, M.-X. Tang, J. V. Hajnal, D. J Wells, and R. J. Eckersley, "Influence of needle gauge on in vivo ultrasound and microbubble-mediated gene transfection," Ultrasound Med. Biol., vol. 37, no. 9, pp. 1531-1537, 2011. [Online]. Available: http://dx.doi.org/10.1016/j.ultrasmedbio.2011.05.019

[21] T. Barrack and E. Stride, "Microbubble destruction during intravenous administration: A preliminary study," Ultrasound Med. Biol., vol. 35, no. 3, pp. 515-522, 2009.

[22] P. S. Sheeran and P. A. Dayton, "Phase-change contrast agents for imaging and therapy," Current Pharmaceutical Design, vol. 18, no. 15, pp. $2152-2165,2012$.

[23] H. Mulvana, E. Stride, M. Tang, J. V. Hajnal, and R. Eckersley, "Temperature-dependent differences in the nonlinear acoustic behavior of ultrasound contrast agents revealed by high-speed imaging and bulk acoustics," Ultrasound Med. Biol., vol. 37, no. 9, pp. 1509-1517, 2011.

[24] C. Sun, V. Sboros, M. B. Butler, and C. M. Moran, "In vitro acoustic characterization of three phospholipid ultrasound contrast agents from 12 to $43 \mathrm{MHz}$," Ultrasound Med. Biol., vol. 40, no. 3, pp. 541-550, 2014.

[25] A. Bouakaz, N. De Jong, and C. Cachard, "Standard properties of ultrasound contrast agents," Ultrasound Med. Biol., vol. 24, no. 3, pp. 469-472, 1998.

[26] H. Mulvana, R. J. Eckersley, M.-X. Tang, Q. Pankhurst, and E. Stride, "Theoretical and experimental characterisation of magnetic microbubbles," Ultrasound Med. Biol., vol. 38, no. 5, pp. 864-875, 2012.

[27] J. A. Kopechek et al., "Acoustic characterization of echogenic liposomes: Frequency-dependent attenuation and backscatter," J. Acoust. Soc. Amer, vol. 130, no. 5, pp. 3472-3481, 2011.

[28] J. Casey, C. Sennoga, H. Mulvana, J. V. Hajnal, M.-X. Tang, and R. J. Eckersley, "Single bubble acoustic characterization and stability measurement of adherent microbubbles," Ultrasound Med. Biol., vol. 39, no. 5, pp. 903-914, 2013.

[29] D. Thomas, V. Sboros, M. Emmer, H. J. Vos, and N. de Jong, "Optical observations of microbubble oscillation in small tubes," in Proc. IEEE Int. Ultrason. Symp. (IUS), Sep. 2009, pp. 2782-2784.

[30] M. S. Roos, "A technique for the study of acoustic scattering from microparticles," J. Acoust. Soc. Amer., vol. 83, no. 2, pp. 770-776, 1988.

[31] R. A. Roy and R. E. Apfel, "Mechanical characterization of microparticles by scattered ultrasound," J. Acoust. Soc. Amer., vol. 87, no. 6, pp. 2332-2341, 1990.

[32] E. C. Everbach, R. A. Roy, and J. T. Flaherty, "Quantification of submicron DDFP gas bubbles using $30 \mathrm{MHz}$ backscattered ultrasound," J. Acoust. Soc. Amer., vol. 110, no. 5, p. 2615, 2001.

[33] V. Sboros, S. D. Pye, C. A. MacDonald, J. Gomatam, C. M. Moran, and W. N. McDicken, "Absolute measurement of ultrasonic backscatter from single microbubbles," Ultrasound Med. Biol., vol. 31, no. 8, pp. 1063-1072, 2005.

[34] G. Renaud, J. G. Bosch, A. F. W. Van Der Steen, and N. De Jong, "Low-amplitude non-linear volume vibrations of single microbubbles measured with an "acoustical camera," Ultrasound Med. Biol., vol. 40, no. 6, pp. 1282-1295, 2014.

[35] Y. Luan et al., "Lipid shedding from single oscillating microbubbles," Ultrasound Med. Biol., vol. 40, no. 8, pp. 1834-1846, 2014.

[36] J. Tu, J. Guan, Y. Qiu, and T. J. Matula, "Estimating the shell parameters of SonoVue microbubbles using light scattering," J. Acoust. Soc. Amer., vol. 126, no. 6, pp. 2954-2962, 2009.

[37] P. Rademeyer, D. Carugo, J. Y. Lee, and E. Stride, "Microfluidic system for high throughput characterisation of echogenic particles," Lab Chip, vol. 15 , no. 2, pp. 417-428, 2015.

[38] N. de Jong, M. Emmer, A. van Wamel, and M. Versluis, "Ultrasonic characterization of ultrasound contrast agents," Med. Biol. Eng. Comput., vol. 47, no. 8, pp. 861-873, 2009.
[39] E. C. Gelderblom et al., "Brandaris 128 ultra-high-speed imaging facility: 10 years of operation, updates, and enhanced features," Rev. Sci. Instrum., vol. 83, no. 10, p. 103706, 2012.

[40] J. E. Chomas, P. A. Dayton, D. May, J. Allen, A. Klibanov, and K. Ferrara, "Optical observation of contrast agent destruction," Appl. Phys. Lett., vol. 77, no. 7, pp. 1056-1058, 2000.

[41] C. F. Caskey, S. M. Stieger, S. Qin, P. A. Dayton, and K. W. Ferrara, "Direct observations of ultrasound microbubble contrast agent interaction with the microvessel wall," J. Acoust. Soc. Amer. vol. 122 , no. 2 , pp. 1191-1200, 2007.

[42] P. Krehl, S. Engemann, C. Rembe, and P. E. Hofer, "High-speed visualization, a powerful diagnostic tool for microactuators-Retrospect and prospect," Microsyst. Technol., vol. 5, no. 3, pp. 113-132, 1999.

[43] H. Chen, "Ultra-high speed optical imaging of ultrasound-activated microbubbles in mesenteric microvessels," Ultrasound Med Biol., vol. 37, no. 12, pp. 2139-2148, Dec. 2011.

[44] P. A. Dayton et al., "Optical and acoustical dynamics of microbubble contrast agents inside neutrophils," Biophys. J., vol. 80, no. 3, pp. 1547-1556, 2001.

[45] C. T. Chin et al., "Brandaris 128: A digital 25 million frames per second camera with 128 highly sensitive frames," Rev. Sci. Instrum., vol. 74, no. 12, pp. 5026-5034, 2003.

[46] X. Chen, J. Wang, M. Versluis, N. de Jong, and F. S. Villanueva, "Ultra-fast bright field and fluorescence imaging of the dynamics of micrometer-sized objects," Rev. Sci. Instrum., vol. 84, no. 6, p. 063701, 2013.

[47] M. Emmer, A. van Wamel, D. E. Goertz, and N. de Jong, "The onset of microbubble vibration," Ultrasound Med. Biol., vol. 33, no. 6 , pp. 941-949, 2007.

[48] N. de Jong et al., "'Compression-only' behavior of phospholipid-coated contrast bubbles," Ultrasound Med. Biol., vol. 33, no. 4, pp. 653-656, 2007.

[49] M. Versluis et al., "Microbubble shape oscillations excited through ultrasonic parametric driving," Phys. Rev. E, vol. 82, p. 026321, Aug. 2010.

[50] M. A. Borden, D. E. Kruse, C. F. Caskey, S. Zhao, P. A. Dayton, and K. W. Ferrara, "Influence of lipid shell physicochemical properties on ultrasound-induced microbubble destruction," IEEE Trans. Ultrason., Ferroelect., Freq. Control, vol. 52, no. 11, pp. 1992-2002, Nov. 2005.

[51] J. Viti, R. Mori, F. Guidi, M. Versluis, N. De Jong, and P. Tortoli, "Correspondence-Nonlinear oscillations of deflating bubbles," IEEE Trans. Ultrason., Ferroelect., Freq. Control, vol. 59, no. 12, pp. 2818-2824, Dec. 2012.

[52] E. C. Gelderblom, "Ultra-high-speed fluorescence imaging," M.S. thesis, Dept. Phys. Fluids Group Faculty Sci. Technol., Univ. Twente, Enschede, The Netherlands, 2012.

[53] Y. Luan et al., "Liposome shedding from a vibrating microbubble on nanoseconds timescale," in Proc. IEEE Int. Ultrason. Symp. (IUS), Jul. 2013, pp. 57-60.

[54] J.-P. O'Brien, N. Ovenden, and E. Stride, "Accounting for the stability of microbubbles to multi-pulse excitation using a lipid-shedding model," J. Acoust. Soc. Amer., vol. 130, no. 4, pp. EL180-1-EL180-5, 2011.

[55] O. Shpak, M. Verweij, N. de Jong, and M. Versluis, "Droplets, bubbles and ultrasound interactions," Adv. Experim. Med. Biol., vol. 880, pp. 157-174, 2016.

[56] J. L. Raymond et al., "Impulse response method for characterization of echogenic liposomes," J. Acoust. Soc. Amer, vol. 137, no. 4, pp. 1693-1703, 2015.

[57] N. de Jong, L. Hoff, T. Skotland, and N. Bom, "Absorption and scatter of encapsulated gas filled microspheres: Theoretical considerations and some measurements," Ultrasonics, vol. 30, pp. 95-103, Mar. 1992.

[58] T. G. Leighton, The Acoustic Bubble, 1st ed. London, U.K.: Academic, 1994.

[59] P. Marmottant et al., "A model for large amplitude oscillations of coated bubbles accounting for buckling and rupture," J. Acoust. Soc. Amer. vol. 118 , no. 6 , pp. 3499-3505, 2005.

[60] S. M. van der Meer et al., "Microbubble spectroscopy of ultrasound contrast agents," J. Acoust. Soc. Amer., vol. 121, no. 1, pp. 648-656, 2007.

[61] Y. Luan et al., "Acoustical properties of individual liposome-loaded microbubbles," Ultrasound Med. Biol., vol. 38, no. 12, pp. 2174-2185, 2012.

[62] T. van Rooij et al., "Non-linear response and viscoelastic properties of lipid-coated microbubbles: DSPC versus DPPC," Ultrasound Med. Biol., vol. 41, pp. 1432-1445, May 2012. 
[63] T. Faez, M. Emmer, M. Docter, J. Sijl, M. Versluis, and N. de Jong, "Characterizing the subharmonic response of phospholipidcoated microbubbles for carotid imaging," Ultrasound Med. Biol., vol. 37, no. 6, pp. 958-970, 2011.

[64] T. Faez, I. Skachkov, M. Versluis, K. Kooiman, and N. de Jong, "In vivo characterization of ultrasound contrast agents: Microbubble spectroscopy in a chicken embryo," Ultrasound Med. Biol., vol. 38, no. 9, pp. 1608-1617, 2012.

[65] J. E. Leeman et al., "Effect of acoustic conditions on microbubblemediated microvascular sonothrombolysis," Ultrasound Med. Biol., vol. 38, no. 9, pp. 1589-1598, 2012.

[66] B. D. Meijering, R. H. Henning, W. H. Van Gilst, I. Gavrilović, A. Van Wamel, and L. E. Deelman, "Optimization of ultrasound and microbubbles targeted gene delivery to cultured primary endothelial cells," J. Drug Targeting, vol. 15, no. 10, pp. 664-671, 2007.

[67] K. Hynynen, N. McDannold, N. Vykhodtseva, and F. A. Jolesz, "Noninvasive MR imaging-guided focal opening of the blood-brain barrier in rabbits," Radiology, vol. 220, no. 3, pp. 640-646, Sep. 2001.

[68] A. Bouakaz, M. Versluis, and N. de Jong, "High-speed optical observations of contrast agent destruction," Ultrasound Med. Biol., vol. 31, no. 3, pp. 391-399, 2005.

[69] F. Guidi, H. J. Vos, R. Mori, N. D. Jong, and P. Tortoli, "Microbubble characterization through acoustically induced deflation," IEEE Trans. Ultrason., Ferroelect., Freq. Control, vol. 57, no. 1, pp. 193-202, Jan. 2010.

[70] D. H. Thomas, M. Butler, N. Pelekasis, T. Anderson, E. Stride, and V. Sboros, "The acoustic signature of decaying resonant phospholipid microbubbles," Phys. Med. Biol., vol. 58, no. 3, pp. 589-599, 2013.

[71] J. E. Chomas, P. Dayton, D. May, and K. Ferrara, "Threshold of fragmentation for ultrasonic contrast agents," J. Biomed. Opt., vol. 6, no. 2, pp. 141-150, 2001.

[72] J. E. Chomas, P. Dayton, J. Allen, K. Morgan, and K. W. Ferrara, "Mechanisms of contrast agent destruction," IEEE Trans. Ultrason., Ferroelect., Freq. Control, vol. 48, no. 1, pp. 232-248, Jan. 2001.

[73] T. J. A. Kokhuis, Y. Luan, F. Mastik, R. H. S. H. Beurskens, M. Versluis, and N. de Jong, "Segmented high speed imaging of vibrating microbubbles during long ultrasound pulses," in Proc. IEEE Int. Ultrason. Symp. (IUS), Oct. 2012, pp. 1343-1346.

[74] I. Skachkov, "Microbubbles for molecular imaging and drug delivery," Ph.D. dissertation, Dept. Secretary Biomed. Eng., Erasmus Univ. Rotterdam, Rotterdam, The Netherlands, 2016.

[75] D. J. Cox and J. L. Thomas, "Rapid shrinkage of lipid-coated bubbles in pulsed ultrasound," Ultrasound Med. Biol., vol. 39, no. 3, pp. 466-474, Mr. 2013.

[76] D. H. Thomas et al., "The 'quasi-stable' lipid shelled microbubble in response to consecutive ultrasound pulses," Appl. Phys. Lett., vol. 101, no. 7, p. 071601, 2012.

[77] J. L. Raymond et al., "Broadband attenuation measurements of phospholipid-shelled ultrasound contrast agents," Ultrasound Med. Biol., vol. 40, no. 2, pp. 410-421, Feb. 2014.

[78] H. J. Vos, B. Dollet, M. Versluis, and N. de Jong, "Nonspherical shape oscillations of coated microbubbles in contact with a wall," Ultrasound Med. Biol., vol. 37, no. 6, pp. 935-948, Jun. 2011.

[79] C. C. Church, "The effects of an elastic solid surface layer on the radial pulsations of gas bubbles," J. Acoust. Soc. Amer., vol. 97, no. 3, pp. 1510-1521, 1995.

[80] C. Harfield, C. R. Fury, G. Memoli, P. Jones, N. Ovenden, and E. Stride, "Analysis of the uncertainty in microbubble characterization," Ultrasound Med. Biol., vol. 42, no. 6, pp. 1412-1418, Jun. 2016.

[81] D. H. Kim, M. J. Costello, P. B. Duncan, and D. Needham, "Mechanical properties and microstructure of polycrystalline phospholipid monolayer shells: Novel solid microparticles," Langmuir, vol. 19, no. 20, pp. 8455-8466, 2003.

[82] V. Sboros et al., "Nanomechanical probing of microbubbles using the atomic force microscope," Ultrasonics, vol. 46, no. 4, pp. 349-354, Nov. 2007.

[83] M. A. Borden et al., "Lateral phase separation in lipid-coated microbubbles," Langmuir, vol. 22, no. 9, pp. 4291-4297, 2006.

[84] J. Owen and E. Stride, "Technique for the characterization of phospholipid microbubbles coatings by transmission electron microscopy," Ultrasound Med. Biol., vol. 41, no. 12, pp. 3253-3258, Dec. 2015.

[85] M. E. Nipper, M. Dakanali, E. Theodorakis, and M. A. Haidekker, "Detection of liposome membrane viscosity perturbations with ratiometric molecular rotors," Biochimie, vol. 93, no. 6, pp. 988-994, Jun. 2011.
[86] N. A. Hosny et al., "Mapping microbubble viscosity using fluorescence lifetime imaging of molecular rotors," Proc. Nat. Acad. Sci. USA, vol. 110, no. 23, pp. 9225-9230, 2013.

[87] K. Kooiman et al., "Lipid distribution and viscosity of coated microbubbles," in Proc. IEEE Ultrason. Symp. (IUS), Oct. 2010, pp. 900-903.

[88] S. W. Hell and E. H. K. Stelzer, "Fundamental improvement of resolution with a 4Pi-confocal fluorescence microscope using twophoton excitation," Opt. Commun., vol. 93, nos. 5-6, pp. 277-282, Oct. 1992.

[89] T. Parasassi, G. De Stasio, G. Ravagnan, R. M. Rusch, and E. Gratton, "Quantitation of lipid phases in phospholipid vesicles by the generalized polarization of laurdan fluorescence," Biophys. J., vol. 60, no. 1, pp. 179-189, 1991.

[90] W. He et al., "Microbubbles with surface coated by superparamagnetic iron oxide nanoparticles," Mater. Lett., vol. 68, pp. 64-67, Feb. 2012.

[91] F. Yang, Y. Li, Z. Chen, Y. Zhang, J. Wu, and N. Gu, "Superparamagnetic iron oxide nanoparticle-embedded encapsulated microbubbles as dual contrast agents of magnetic resonance and ultrasound imaging," Biomaterials, vol. 30, nos. 23-24, pp. 3882-3890, Aug. 2009.

[92] D. Vlaskou et al., "Magnetic and acoustically active lipospheres for magnetically targeted nucleic acid delivery," Adv. Funct. Mater, vol. 20, no. 22 , pp. 3881-3894, Nov. 2010.

[93] K. E. Hitchcock et al., "Ultrasound-enhanced delivery of targeted echogenic liposomes in a novel ex vivo mouse aorta model," J. Controlled Release, vol. 144, no. 3, pp. 288-295, Jun. 2010.

[94] R. Abdalkader et al., "Evaluation of the potential of doxorubicin loaded microbubbles as a theranostic modality using a murine tumor model," Acta Biomater., vol. 19, pp. 112-118, Jun. 2015.

[95] S. R. Sirsi, C. Fung, S. Garg, M. Y. Tianning, P. A. Mountford, and M. A. Borden, "Lung surfactant microbubbles increase lipophilic drug payload for ultrasound-targeted delivery," Theranostics, vol. 3, no. 6, pp. 409-419, 2013.

[96] M. C. Cochran, J. Eisenbrey, R. O. Ouma, M. Soulen, and M. A. Wheatley, "Doxorubicin and paclitaxel loaded microbubbles for ultrasound triggered drug delivery," Int. J. Pharmaceutics, vol. 414, nos. 1-2, pp. 161-170, Jul. 2011.

[97] C. Pu et al., "Ultrasound-mediated destruction of LHRHa-targeted and paclitaxel-loaded lipid microbubbles for the treatment of intraperitoneal ovarian cancer xenografts," Molecular Pharmaceutics, vol. 11, no. 1, pp. 49-58, 2014

[98] A. I. Ivanov et al., "Cisplatin binding sites on human albumin," J. Biol. Chem., vol. 273, pp. 14721-14730, Jun. 1998.

[99] Y. Lei, N. Nosoudi, and N. Vyavahare, "Targeted chelation therapy with EDTA-loaded albumin nanoparticles regresses arterial calcification without causing systemic side effects," J. Controlled Release, vol. 196, pp. 79-86, Dec. 2014.

[100] G. Stehle, A. Wunder, H. H. Schrenk, G. Hartung, D. L. Heene, and H. Sinn, "Albumin-based drug carriers: Comparison between serum albumins of different species on pharmacokinetics and tumor uptake of the conjugate," Anticancer Drugs, vol. 10, no. 8, pp. 785-790, 1999.

[101] B. D. Butler and B. A. Hills, "The lung as a filter for microbubbles," J. Appl. Physiol., vol. 47, no. 3, pp. 537-543, 1979.

[102] M. J. K. Blomley et al., "Stimulated acoustic emission to image a late liver and spleen-specific phase of Levovist in normal volunteers and patients with and without liver disease," Ultrasound Med. Biol., vol. 25 , no. 9, pp. 1341-1352, Nov. 1999.

[103] A. K. P. Lim, N. Patel, R. J. Eckersley, S. D. Taylor-Robinson, D. O. Cosgrove, and M. J. K. Blomley, "Evidence for spleen-specific uptake of a microbubble contrast agent: A quantitative study in healthy volunteers," Radiology, vol. 231, no. 3, pp. 785-788, Jun. 2004.

[104] M. J. Blomley et al., "Do different types of liver lesions differ in their uptake of the microbubble contrast agent SH U 508A in the late liver phase? Early experience," Radiology, vol. 220, no. 3, pp. 661-667, Sep. 2001.

[105] T. H. Bryant et al., "Improved characterization of liver lesions with liver-phase uptake of liver-specific microbubbles: Prospective multicenter study," Radiology, vol. 232, no. 3, pp. 799-809, Sep. 2004.

[106] M. Seo, I. Gorelikov, R. Williams, and N. Matsuura, "Microfluidic assembly of monodisperse, nanoparticle-incorporated perfluorocarbon microbubbles for medical imaging and therapy," Langmuir, vol. 26, no. 17 , pp. $13855-13860,2010$.

[107] L. Duan et al., "Controlled assembly of magnetic nanoparticles on microbubbles for multimodal imaging," Soft Matter, vol. 11, no. 27, pp. 5492-5500, 2015. 
[108] S. Tinkov, G. Winter, C. Coester, and R. Bekeredjian, "New doxorubicin-loaded phospholipid microbubbles for targeted tumor therapy: Part I-Formulation development and in-vitro characterization," J. Controlled Release, vol. 143, no. 1, pp. 143-150, Apr. 2010.

[109] F. Ja'afar, C. H. Leow, V. Garbin, C. A. Sennoga, M.-X. Tang, and J. M. Seddon, "Surface charge measurement of SonoVue, Definity and Optison: A comparison of laser doppler electrophoresis and micro-electrophoresis," Ultrasound Med. Biol., vol. 41, no. 11, pp. 2990-3000, Nov. 2015.

[110] J. R. Griffiths, "Are cancer cells acidic?" Brit. J. Cancer, vol. 64, no. 3, pp. 425-427, 1991.

[111] I. F. Tannock and D. Rotin, "Acid pH in tumors and its potential for therapeutic exploitation," Cancer Res., vol. 49, no. 16, pp. 4373-4384, 1989.

[112] L. E. Gerweck and K. Seetharaman, "Cellular $\mathrm{pH}$ gradient in tumor versus normal tissue: Potential exploitation for the treatment of cancer," Cancer Res., vol. 56, no. 6, pp. 1194-1198, Mar. 1996.

[113] A. Kheirolomoom et al., "Acoustically-active microbubbles conjugated to liposomes: Characterization of a proposed drug delivery vehicle," J. Controlled Release, vol. 118, no. 3, pp. 275-284, Apr. 2007.

[114] S. R. Sirsi and M. A. Borden, "Microbubble compositions, properties and biomedical applications," Bubble Sci., Eng. Technol., vol. 1, nos. 1-2, pp. 3-17, 2009.

[115] E. Fahrländer, S. Schelhaas, A. H. Jacobs, and K. Langer, "PEGylated human serum albumin (HSA) nanoparticles: Preparation, characterization and quantification of the PEGylation extent," Nanotechnology, vol. 26, no. 14, p. 145103, 2015.

[116] W. W. Christie, Gas Chromatography and Lipids: A Practical Guide. Dundee, Scotland: Oily Press Ltd., 1989.

[117] W. W. Christie and X. Han, Lipid Analysis: Isolation, Separation, Identification and Lipidomic Analysis, 4th ed. Dundee, Scotland: Oily Press Ltd., 2010.

[118] A. Derenne, O. Vandersleyen, and E. Goormaghtigh, "Lipid quantification method using FTIR spectroscopy applied on cancer cell extracts," Biochim. Biophys. Acta, vol. 1841, no. 8, pp. 1200-1209, Aug. 2014.

[119] E. Hvattum, S. Uran, A. G. Sandbæk, A. Å. Karlsson, and T. Skotland, "Quantification of phosphatidylserine, phosphatidic acid and free fatty acids in an ultrasound contrast agent by normalphase high-performance liquid chromatography with evaporative light scattering detection," J. Pharmaceutical Biomed. Anal., vol. 42, no. 4 , pp. 506-512, Oct. 2006

[120] H. P. Nissen and H. W. Kreysel, "The use of HPLC for the determination of lipids in biological materials," Chromatographia, vol. 30, no. 11, pp. 686-690, Dec. 1990

[121] J.-M. Escoffre and A. Bouakaz, Eds., Therapeutic Ultrasound: From Biophysics Concepts to Clinical Applications. Switzerland: Springer, 2016.

[122] M. González et al., "Interaction of biotin with streptavidin: Thermostability and conformational changes upon binding," J. Biol. Chem., vol. 272, pp. 11288-11294, Apr. 1997.

[123] Thermo Fisher Scientific Inc. (2009). Avidin-Biotin Technical Handbook. [Online]. Available: https://tools.thermofisher.com/content/ sfs/brochures/1601675-Avidin-Biotin-Handbook.pdf

[124] J. S.-M. Yeh et al., "A targeting microbubble for ultrasound molecular imaging," PLoS ONE, vol. 10, no. 7, p. e0129681, 2015.

[125] S. P. Wrenn, M. Mleczko, and G. Schmitz, "Phospholipid-stabilized microbubbles: Influence of shell chemistry on cavitation threshold and binding to giant uni-lamellar vesicles," Appl. Acoust., vol. 70, no. 10, pp. 1313-1322, Oct. 2009

[126] J. Zhang et al., "Preparation and characterization of luteinisinghormone releasing hormone nanoliposomal microbubbles specifically targeting ovarian cancer cells in vitro," Molecular Med. Rep., vol. 10, pp. 567-571, May 2014

[127] F. Petronzelli et al., "Therapeutic use of avidin is not hampered by antiavidin antibodies in humans," Cancer Biotherapy Radiopharmaceuticals, vol. 25, no. 5, pp. 563-570, Oct. 2010.

[128] M. Chinol et al., "Biochemical modifications of avidin improve pharmacokinetics and biodistribution, and reduce immunogenicity," Brit. J. Cancer, vol. 78, pp. 189-197, Jul. 1998.

[129] F. Petronzelli et al., "Preclinical pharmacology and safety of a novel avidin derivative for tissue-targeted delivery of radiolabelled biotin," Basic Clin. Pharmacol. Toxicol., vol. 109, no. 36, pp. 145-155, Sep. 2011.
[130] R. H. Batchelor, A. Sarkez, W. G. Cox, and I. Johnson, "Fluorometric assay for quantitation of biotin covalently attached to proteins and nucleic acids," Biotechniques, vol. 43, no. 4, pp. 503-507, Oct. 2007.

[131] Pierce Fluorescence Biotin Quantitation Kit, accessed on Mar. 31, 2016. [Online]. Available: https://www.thermofisher.com/ order/catalog/product $/ 46610$

[132] Y.-R. Liou, Y.-H. Wang, C.-Y. Lee, and P.-C. Li, "Buoyancy-activated cell sorting using targeted biotinylated albumin microbubbles," PLoS ONE, vol. 10, no. 5, p. e0125036, 2015.

[133] A. M. Takalkar, A. L. Klibanov, J. J. Rychak, J. R. Lindner, and K. Ley, "Binding and detachment dynamics of microbubbles targeted to P-selectin under controlled shear flow," J. Controlled Release, vol. 96, no. 3, pp. 473-482, May 2004.

[134] M. A. Nakatsuka, M. J. Hsu, S. C. Esener, J. N. Cha, and A. P. Goodwin, "DNA-coated microbubbles with biochemically tunable ultrasound contrast activity," Adv. Mater, vol. 23, no. 42, pp. 4908-4912, Nov. 2011.

[135] C. W. Burke, Y.-H. J. Hsiang, E. Alexander, IV, A. L. Kilbanov, and R. J. Price, "Covalently linking poly(lactic-co-glycolic acid) nanoparticles to microbubbles before intravenous injection improves their ultrasound-targeted delivery to skeletal muscle," Small, vol. 7, no. 9, pp. 1227-1235, May 2011.

[136] Q. P. Lei et al., "Quantification of residual EDU ( $N$-ethyl- $N$ ' (dimethylaminopropyl) carbodiimide (EDC) hydrolyzed urea derivative) and other residual by LC-MS/MS," J. Chromatogr. B, vol. 813, nos. 1-2, pp. 103-112, Dec. 2004.

[137] B. S. Jacobson and K. R. Fairman, "A colorimetric assay for carbodiimides commonly used in peptide synthesis and carboxyl group modification," Anal. Biochem., vol. 106, no. 1, pp. 114-117, Jul. 1980.

[138] M. Wilchek, T. Miron, and J. Kohn, "A highly sensitive colorimetric method for the determination of carbodiimides," Anal. Biochem., vol. 114, no. 2, pp. 419-421, Jul. 1981.

[139] T. Miron and M. Wilchek, "A spectrophotometric assay for soluble and immobilized $N$-hydroxysuccinimide esters," Anal. Biochem., vol. 126 , no. 2, pp. 433-435, Nov. 1982.

[140] A. Gao et al., "Facile spectrophotometric assay of molar equivalents of $N$-hydroxysuccinimide esters of monomethoxyl poly-(ethylene glycol) derivatives," Chem. Central J., vol. 6, pp. 1-9, Dec. 2012.

[141] O. Klykov and M. G. Weller, "Quantification of $N$-hydroxysuccinimide and $N$-hydroxysulfosuccinimide by hydrophilic interaction chromatography (HILIC)," Anal. Methods, vol. 7, pp. 6443-6448, Jun. 2015.

[142] H. J. Gruber and H. Schindler, "External surface and lamellarity of lipid vesicles: A practice-oriented set of assay methods," Biochim. Biophys. Acta, vol. 1189, no. 2, pp. 212-224, Jan. 1994.

[143] H. Engel, E. Rondeau, E. J. Windhab, and P. Walde, "External surface area determination of lipid vesicles using trinitrobenzene sulfonate and ultraviolet/visible spectrophotometry," Anal. Biochem., vol. 442, no. 2, pp. 262-271, Nov. 2013.

[144] F. Everaerts, M. Torrianni, M. Hendriks, and J. Feijen, "Quantification of carboxyl groups in carbodiimide cross-linked collagen sponges," J. Biomed. Mater. Res. A, vol. 83A, no. 4, pp. 1176-1183, 2007.

[145] P. S. Mukherjee, K. H. DeSilva, and H. T. Karnes, "5-bromomethyl fluorescein (5-BMF) for derivatization of carboxyl containing analytes for use with laser-induced fluorescence detection," Pharmaceutical Res., vol. 12, no. 6, pp. 930-936, 1995.

[146] R. Jue, J. M. Lambert, L. R. Pierce, and R. R. Traut, "Addition of sulfhydryl groups of Escherichia coli ribosomes by protein modification with 2-iminothiolane (methyl 4-mercaptobutyrimidate)," Biochemistry, vol. 17, no. 25, pp. 5399-5406, 1978.

[147] M. W. Hougland and J. M. Luethke, "Quantitation of sulfhydryl groups on erythrocytes in polycythemia vera," Histochemistry, vol. 76, no. 1 , pp. 57-60, 1982.

[148] F. W. Stratman, A. A. Hochberg, R. N. Zahlten, and H. P. Morris, "Sulfhydryl group quantitation of hepatoma and liver microsomal fractions," Cancer Res., vol. 35, no. 6, pp. 1476-1484, 1975.

[149] J. Sedlak and R. H. Lindsay, "Estimation of total, protein-bound, and nonprotein sulfhydryl groups in tissue with Ellman's reagent," Anal. Biochem., vol. 25, no. 1, pp. 192-205, 1968.

[150] Fluorometric Thiol Assay Kit, accessed on Mar. 31, 2016. [Online] Available: http://www.sigmaaldrich.com/catalog/product/sigma/mak151

[151] Measure-IT Thiol Assay Kit. [Online]. Available: https://www. thermofisher.com/order/catalog/product/M30550 
[152] S. R. Sirsi et al., "Polyplex-microbubble hybrids for ultrasound-guided plasmid DNA delivery to solid tumors," J. Controlled Release, vol. 157, no. 2, pp. 224-234, 2012.

[153] G. Shi et al., "Isolation of rare tumor cells from blood cells with buoyant immuno-microbubbles," PLoS ONE, vol. 8, no. 3, p. e58017, 2013

[154] G. Shi, W. Cui, R. Mukthavaram, Y.-T. Liu, and D. Simberg, "Binding and isolation of tumor cells in biological media with perfluorocarbon microbubbles," Methods, vol. 64, pp. 102-107, Dec. 2013.

[155] A. J. van der Vlies, C. P. O'Neil, U. Hasegawa, N. Hammond, and J. A. Hubbell, "Synthesis of pyridyl disulfide-functionalized nanoparticles for conjugating thiol-containing small molecules, peptides, and proteins," Bioconjugate Chem., vol. 21, no. 4, pp. 653-662, 2010.

[156] Y. Lu et al., "Assessment of atherosclerotic plaques in the rabbit abdominal aorta with interleukin-8 monoclonal antibody-targeted ultrasound microbubbles," Molecular Biol. Rep., vol. 40, no. 4, pp. 3083-3092, 2013.

[157] Y. I. Yoon et al., "Ultrasound-mediated gene and drug delivery using a microbubble-liposome particle system," Theranostics, vol. 4, no. 11, pp. 1133-1144, 2014.

[158] Maleimide Quantification Assay Kit, accessed on Mar. 31, 2016. [Online]. Available: http://www.abcam.com/ maleimide-quantification-assay-kit-fluorometric-ab112141.html

[159] R. Singh, "A sensitive assay for maleimide groups," Bioconjugate Chem., vol. 5, no. 4, pp. 348-351, 1994.

[160] J. Carlsson, H. Drevin, and R. Axén, "Protein thiolation and reversible protein-protein conjugation. $N$-succinimidyl 3-(2-pyridyldithio)propionate, a new heterobifunctional reagent," Biochem. J., vol. 173, no. 3, pp. 723-737, 1978.

[161] G. Ilangovan, H. Li, J. L. Zweier, and P. Kuppusamy, "In vivo measurement of tumor redox environment using EPR spectroscopy," Molecular Cellular Biochem., vol. 234, no. 1, pp. 393-398, 2002.

[162] J. A. Feshitan, C. C. Chen, J. J. Kwan, and M. A. Borden, "Microbubble size isolation by differential centrifugation," J. Colloid Interface Sci., vol. 329, no. 2, pp. 316-324, 2009.

[163] F. Cavalieri et al., "Antimicrobial and biosensing ultrasound-responsive lysozyme-shelled microbubbles," ACS Appl. Mater. Interfaces, vol. 5, no. 2, pp. 464-471, 2013.

[164] S. Melino, M. Zhou, M. Tortora, M. Paci, F. Cavalieri, and M. Ashokkumar, "Molecular properties of lysozyme-microbubbles: Towards the protein and nucleic acid delivery," Amino Acids, vol. 43, no. 2, pp. 885-896, 2012.

[165] F. Cavalieri, M. Ashokkumar, F. Grieser, and F. Caruso, "Ultrasonic synthesis of stable, functional lysozyme microbubbles," Langmuir, vol. 24, no. 18, pp. 10078-10083, 2008.

[166] M. S. Tartis et al., "Dynamic microPET imaging of ultrasound contrast agents and lipid delivery," J. Controlled Release, vol. 131, no. 3, pp. 160-166, 2008.

[167] L. Zhang et al., "Ultrasound-triggered microbubble destruction in combination with cationic lipid microbubbles enhances gene delivery," J. Huazhong Univ. Sci. Technol. [Med. Sci.], vol. 31, no. 1, pp. 39-45, 2011.

[168] H. Yang et al., "Multifunctional PLGA nanobubbles as theranostic agents: Combining doxorubicin and P-gp siRNA Co-delivery into human breast cancer cells and ultrasound cellular imaging," J. Biomed. Nanotechnol., vol. 11, pp. 2124-2136, Dec. 2015.

[169] O. Boussif et al., "A versatile vector for gene and oligonucleotide transfer into cells in culture and in vivo: Polyethylenimine," Proc. Nat. Acad. Sci. USA, vol. 92, pp. 7297-7301, Aug. 1995.

[170] C. M. Panje et al., "Ultrasound-mediated gene delivery with cationic versus neutral microbubbles: Effect of DNA and microbubble dose on in vivo transfection efficiency," Theranostics, vol. 2, no. 11, pp. 1078-1091, 2012.

[171] D. S. Wang et al., "Cationic versus neutral microbubbles for ultrasoundmediated gene delivery in cancer," Radiology, vol. 264, pp. 721-732, Sep. 2012.

[172] W. T. Godbey, K. K. Wu, and A. G. Mikos, "Poly(ethylenimine) and its role in gene delivery," J. Controlled Release, vol. 60, pp. 149-160, Aug. 1999.

[173] Q.-Q. Zhao et al., "N/P ratio significantly influences the transfection efficiency and cytotoxicity of a polyethylenimine/chitosan/DNA complex," Biol. Pharmaceutical Bull., vol. 32, no. 4, pp. 706-710, 2009.
[174] C.-B. Zhang et al., "Enhancement effect of ultrasound-induced microbubble cavitation on branched polyethylenimine-mediated VEGF165 transfection with varied N/P ratio," Ultrasound Med. Biol., vol. 39, no. 1, pp. 161-171, 2013.

[175] S. Tavri, A. Vezeridis, W. Cui, and R. F. Mattrey, "In vivo transfection and detection of gene expression of stem cells preloaded with DNAcarrying microbubbles," Radiology, vol. 276, no. 2, pp. 518-525, 2015.

[176] A. Aitken and M. P. Learmonth, "Protein determination by UV absorption," in The Protein Protocols Handbook, J. M. Walker, Ed. Totowa, NJ, USA: Humana Press, 2002, pp. 3-6.

[177] D. B. Schowalter and S. S. Sommer, "The generation of radiolabeled DNA and RNA probes with polymerase chain reaction," Anal. Biochem., vol. 177, pp. 90-94, Feb. 1989.

[178] C. G. P. Mathew, Radiolabeling of DNA by Nick Translation (Methods in Molecular Biology), vol. 2. Totowa, NJ, USA: Humana Press, 1985 pp. 257-261.

[179] C. G. P. Mathew, "Radiolabeling of DNA by nick translation," in Nucleic Acids, J. M. Walker, Ed. Totowa, NJ, USA: Humana Press, 1984, pp. 257-261.

[180] P. S. Aranda, D. M. LaJoie, and C. L. Jorcyk, "Bleach gel: A simple agarose gel for analyzing RNA quality," Electrophoresis, vol. 33, no. 2, pp. 366-369, 2012.

[181] N. L. Adkins, J. A. Hall, and P. T. Georgel, "The use of quantitative agarose gel electrophoresis for rapid analysis of the integrity of proteinDNA complexes," J. Biochem. Biophys. Methods, vol. 70, no. 5, pp. 721-726, 2007.

[182] J. W. Tweedie and K. M. Stowell, "Quantification of DNA by agarose gel electrophoresis and analysis of the topoisomers of plasmid and M13 DNA following treatment with a restriction endonuclease or DNA topoisomerase I," Biochem. Molecular Biol. Edu., vol. 33, no. 1, pp. 28-33, 2005.

[183] D. Pramanick, J. Forstová, and L. Pivec, "4 M guanidine hydrochloride applied to the isolation of DNA from different sources," FEBS Lett. vol. 62 , no. 1, pp. 81-84, 1976.

[184] Y. Li and R. R. Breaker, "Kinetics of RNA degradation by specific base catalysis of transesterification involving the 2'-hydroxyl group," J. Amer. Chem. Soc., vol. 121, no. 23, pp. 5364-5372, 1999.

[185] N. Rapoport, Z. Gao, and A. Kennedy, "Multifunctional nanoparticles for combining ultrasonic tumor imaging and targeted chemotherapy," J. Nat. Cancer Inst., vol. 99, no. 14, pp. 1095-1106, 2007.

[186] I. Lentacker, B. Geers, J. Demeester, S. C. De Smedt, and N. N. Sanders, "Design and evaluation of doxorubicin-containing microbubbles for ultrasound-triggered doxorubicin delivery: Cytotoxicity and mechanisms involved," Molecular Therapy, vol. 18, no. 1, pp. 101-108, 2009.

[187] J. R. Eisenbrey, M. C. Soulen, and M. A. Wheatley, "Delivery of encapsulated doxorubicin by ultrasound-mediated size reduction of drug-loaded polymer contrast agents," IEEE Trans. Biomed. Eng., vol. 57, no. 1, pp. 24-28, Jan. 2010.

[188] B. Geers, I. Lentacker, N. N. Sanders, J. Demeester, S. Meairs, and S. C. De Smedt, "Self-assembled liposome-loaded microbubbles: The missing link for safe and efficient ultrasound triggered drug-delivery," J. Controlled Release, vol. 152, no. 2, pp. 249-256, 2011.

[189] W.-C. Huang et al., "Tumortropic monocyte-mediated delivery of echogenic polymer bubbles and therapeutic vesicles for chemotherapy of tumor hypoxia," Biomaterials, vol. 71, pp. 71-83, Dec. 2015

[190] C. W. Burke, E. Alexander, IV, K. Timbie, A. L. Kilbanov, and R. J. Price, "Ultrasound-activated agents comprised of 5FU-bearing nanoparticles bonded to microbubbles inhibit solid tumor growth and improve survival," Molecular Therapy, vol. 22, no. 2, pp. 321-328, 2014.

[191] X. Qian, W. Wang, W. Kong, and Y. Chen, "Organic-inorganic hybrid hollow mesoporous organosilica nanoparticles for efficient ultrasoundbased imaging and controlled drug release," J. Nanomater, vol. 2014 Jan. 2014, Art. no. 115.

[192] F. Yan et al., "Paclitaxel-liposome-microbubble complexes as ultrasound-triggered therapeutic drug delivery carriers," J. Controlled Release, vol. 166, no. 3, pp. 246-255, 2013.

[193] C. McEwan, C. Fowley, N. Nomikou, B. McCaughan, A. P. McHale, and J. F. Callan, "Polymeric microbubbles as delivery vehicles for sensitizers in sonodynamic therapy," Langmuir, vol. 30, no. 49, pp. 14926-14930, 2014 
[194] C.-C. Chang, Y.-T. Yang, J.-C. Yang, H.-D. Wu, and T. Tsai, “Absorption and emission spectral shifts of rose bengal associated with DMPC liposomes," Dyes Pigments, vol. 79, no. 2, pp. 170-175, 2008.

[195] S. Lu, J.-Y. Chen, Y. Zhang, J. Ma, P.-N. Wang, and Q. Peng, "Fluorescence detection of protoporphyrin IX in living cells: A comparative study on single- and two-photon excitation," J. Biomed. Opt., vol. 13, p. 024014, Apr. 2008.

[196] Q. Liu, X. Wang, P. Wang, L. Xiao, and Q. Hao, "Comparison between sonodynamic effect with protoporphyrin IX and hematoporphyrin on sarcoma 180," Cancer Chemother. Pharmacol., vol. 60, no. 5, pp. 671-680, 2007.

[197] E. V. Rosca, M. Wright, R. Gonitel, W. Gedroyc, A. D. Miller, and M. Thanou, "Thermosensitive, near-infrared-labeled nanoparticles for topotecan delivery to tumors," Molecular Pharmaceutics, vol. 12, no. 5, pp. 1335-1346, 2015.

[198] J. H. Francis, B. P. Marr, P. Schaiquevich, M. G. Kellick, and D. H. Abramson, "Properties and clinical utility of topotecan fluorescence: Uses for retinoblastoma," Brit. J. Ophthalmol., vol. 99, pp. 1320-1322, Apr. 2015.

[199] P. Mohan and N. Rapoport, "Doxorubicin as a molecular nanotheranostic agent: Effect of doxorubicin encapsulation in micelles or nanoemulsions on the ultrasound-mediated intracellular delivery and nuclear trafficking," Molecular Pharmaceutics, vol. 7, no. 6, pp. 1959-1973, 2010.

[200] H. Y. Fu, H. L. Wu, J. F. Nie, Y. J. Yu, H. Y. Zou, and R. Q. Yu, "Highly sensitive fluorescence quantification of irinotecan in biological fluids with the aid of second-order advantage," Chin. Chem. Lett., vol. 21, no. 2, pp. 1482-1486, 2010.

[201] V. Augey, M. Cociglio, M. Galtier, R. Yearoo, V. Pinsani, and F. Bressolle, "High-performance liquid chromatographic determination of cis-dichlorodiammineplatinum(II) in plasma ultrafiltrate," J. Pharmaceutical Biomed. Anal., vol. 13, no. 9, pp. 1173-1178, 1995.

[202] R. Paroni, C. Arcelloni, E. De Vecchi, I. Fermo, D. Mauri, and R. Colombo, "Plasma mitomycin $\mathrm{C}$ concentrations determined by HPLC coupled to solid-phase extraction," Clin. Chem., vol. 43, no. 4, pp. 615-618, 1997.

[203] J. Ciccolini, C. Mercier, M.-F. Blachon, R. Favre, A. Durand, and B. Lacarelle, "A simple and rapid high-performance liquid chromatographic (HPLC) method for 5-fluorouracil (5-FU) assay in plasma and possible detection of patients with impaired dihydropyrimidine dehydrogenase (DPD) activity," J. Clin. Pharmacy Therapeutics, vol. 29, no. 4, pp. 307-315, 2004.

[204] H. Wu, Q. Song, G. Ran, X. Lu, and B. Xu, "Recent developments in the detection of singlet oxygen with molecular spectroscopic methods," TrAC Trends Anal. Chem., vol. 30, no. 1, pp. 133-141, 2011.

[205] É. Hideg, C. Barta, T. Kálai, I. Vass, K. Hideg, and K. Asada, "Detection of singlet oxygen and superoxide with fluorescent sensors in leaves under stress by photoinhibition or UV radiation," Plant Cell Physiol., vol. 43, no. 10, pp. 1154-1164, 2002.

[206] X. Wang, S. Guo, S. Lu, J. Zhou, J. Li, and S. Xia, "Ultrasoundinduced release of GDNF from lipid coated microbubbles injected into striatum reduces hypoxic-ischemic injury in neonatal rats," Brain Res. Bull., vol. 88, no. 5, pp. 495-500, 2012.

[207] X. Wang et al., "Intracerebral administration of ultrasound-induced dissolution of lipid-coated GDNF microbubbles provides neuroprotection in a rat model of Parkinson's disease," Brain Res. Bull., vol. 103, pp. 60-65, Apr. 2014.

[208] Q. T. H. Shubhra et al., "Co-encapsulation of human serum albumin and superparamagnetic iron oxide in PLGA nanoparticles: Part I. Effect of process variables on the mean size," J. Microencapsulation, vol. 31, no. 2, pp. 147-155, 2014.

[209] J. E. Noble and M. J. A. Bailey, "Quantitation of protein," in Guide to Protein Purification (Methods in Enzymology), vol. 463, R. B. Richard and P. D. Murray, Eds. New York, NY, USA: Academic, 2009, ch. 8, pp. 73-95.

[210] C. Dauly, D. H. Perlman, C. E. Costello, and M. E. McComb, "Protein separation and characterization by np-RP-HPLC followed by intact MALDI-TOF mass spectrometry and peptide mass mapping analyses," J. Proteome Res., vol. 5, no. 7, pp. 1688-1700, 2006

[211] E. C. Nice and M.-I. Aguilar, "Micropreparative HPLC of peptides and proteins," in HPLC of Peptides and Proteins: Methods and Protocols, M.-I. Aguilar, Ed. New York, NY, USA: Springer, 2004, pp. $165-175$.
[212] T. J. A. Kokhuis et al., "Intravital microscopy of localized stem cell delivery using microbubbles and acoustic radiation force," Biotechnol. Bioeng., vol. 112, no. 1, pp. 220-227, 2015.

[213] M. Kaya et al., "Acoustic radiation force for vascular cell therapy: In vitro validation," Ultrasound Med. Biol., vol. 38, no. 11, pp. 1989-1997, 2012.

[214] T. G. Leighton, "The freely-oscillating bubble," in The Acoustic Bubble. New York, NY, USA: Academic, 1994, ch. 3, pp. 129-286.

[215] K. Kooiman et al., "DSPC or DPPC as main shell component influences ligand distribution and binding area of lipid-coated targeted microbubbles," Eur. J. Lipid Sci. Technol., vol. 116, no. 9, pp. 1217-1227, 2014.

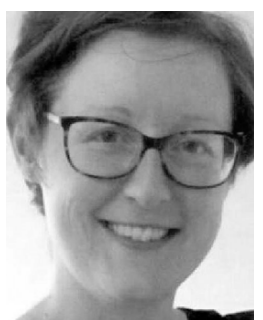

Helen Mulvana received the B.Eng. (Hons.) degree in mechanical engineering from the University of Glasgow, Glasgow, U.K., in 2001, and the Dr. Eng. degree in medical devices from the University of Strathclyde, Glasgow, in 2009.

She joined the industry as a Mechanical Design Engineer and a Project Leader. She was a Postdoctoral Research Assistant at the Imaging Sciences Department, Imperial College London, London, U.K. She joined the School of Engineering, University of Glasgow, as a Lord Kelvin Adam Research Fellow in 2012. Her research focuses on the use of ultrasound and ultrasound contrast agents to improve the diagnosis and treatment of cancer. Her research is funded by the Royal Society, The Carnegie Trust, and Bowel and Cancer Research, U.K. Her current interests include improving the understanding of how ultrasound and microbubbles interact with tissues and cells and developing new imaging methods to aid in the accuracy and availability of ultrasound diagnostic information to clinicians in the pursuit of personalized cancer treatment.

Dr. Mulvana is an Associate Editor of the IEEE TRANSACTIONS ON Ultrasonics, Ferroelectrics, AND FreQuency Control and is on the Technical Program Committee of the IEEE International Ultrasonics Symposium. She is Chartered through the Institute of Mechanical Engineers.

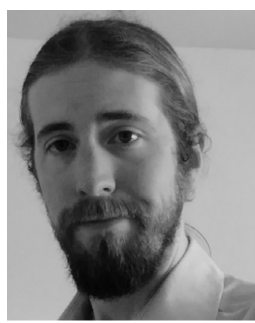

Richard J. Browning received the M.Biochem. degree (Hons.) from the University of Bath, Bath, U.K., in 2007, and the Ph.D. degree in biophysics from Imperial College London, London, U.K., in 2012.

He was with Imperial College London as a Postdoctoral Research Associate in 2012, and with King's College London, London, in 2013, for a second postdoctoral research position. In 2014, he joined the BUBBL Group at the University of Oxford, Oxford, U.K. He has been involved in optimizing UCA parameters, UCA production techniques, and drug delivery by ultrasound to tumors and the heart. His current research interests include the use of ultrasound to mediate delivery of drugs or genetic material by developing novel therapy-based contrast agents (UCAs). 


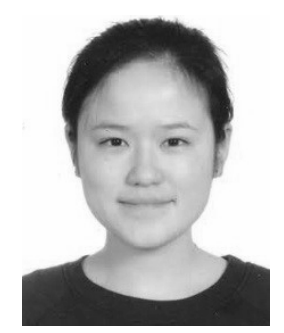

Ying Luan was born in Nanjing, China, in 1985. She received the B.S. degree in biomedical engineering from Southeast University, Nanjing, in 2007, the master's degree in biomedical engineering from the Chalmers University of Technology, Gothenburg, Sweden, in 2009, and the Ph.D. degree in ultrasound contrast agents for drug delivery applications from Erasmus University Rotterdam, Rotterdam, The Netherlands, in 2014.

Since 2015, she has been a Postdoctoral Research Associate with the Department of Imaging Science and Biomedical Engineering, King's College London, London, U.K. Her current research interests include biomedical ultrasound, ultrasound imaging reconstruction, and ultrasound contrast agent for the application of imaging and drug delivery.

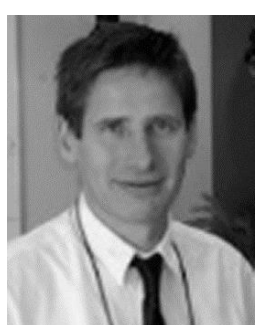

Nico de Jong was born in Nieuwerkerk aan den Ijssel, The Netherlands, in 1954. He received the B.Sc. and M.Sc. degrees in physics from the Delft University of Technology, Delft, The Netherlands, in 1977 and 1978, respectively, and the Ph.D. degree in acoustic properties of ultrasound contrast agents from Erasmus University Rotterdam, Rotterdam, The Netherlands, in 1993.

Since 1980, he has been a Staff Member of the Thoraxcenter of the Erasmus University Medical Center (Erasmus MC), Rotterdam. In 2003, he became a part-time Professor with the Physics of Fluids group headed by Prof. D. Lohse at the University of Twente, Enschede, The Netherlands. Since 2011, he has been a Professor of Molecular Ultrasonic Imaging and Therapy with Erasmus MC and the Technical University of Delft, Delft. He teaches at technical universities and at Erasmus MC. Over the past 5 years, he has given more than 30 invited lectures and numerous scientific presentations for international industries. He has been a promotor of $15 \mathrm{Ph} . \mathrm{D}$. students and is currently supervising nine Ph.D. students.

Dr. de Jong is the organizer of the annual European Symposium on Ultrasound Contrast Imaging, held in Rotterdam, and attended by approximately 175 scientists from universities and industries all over the world. $\mathrm{He}$ is on the Safety Committee of the World Federation of Ultrasound in Medicine and Biology, an Associate Editor of Ultrasound in Medicine and Biology, and has been a Guest Editor of special issues of different journals.

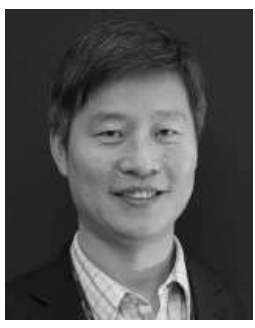

Meng-Xing Tang was a Postdoctoral Researcher and a Departmental Lecturer with the Department of Engineering Science, University of Oxford, Oxford, U.K., from 2002 to 2006. He joined the Department of Bioengineering, Imperial College, London, U.K., as a University Lecturer (Assistant Professor) in 2006, became a Senior Lecturer (Associate Professor) in 2011, and a Reader in 2015. After joining Imperial College, he founded and currently leads the Ultrasound Laboratory for Imaging and Sensing (ULIS, previously known as the Ultrasound Imaging Group). He has authored more than 60 peer reviewed journal papers. In particular, he is involved in developing and applying new ultrasound imaging techniques of high resolution (both spatial and temporal) and contrast. His research has been funded by U.K. EPSRC, Cancer Research U.K., the Wellcome Trust, the Royal Society, and the Bagrit Foundation. His current research interests include developing new imaging and image analysis techniques using ultrasound and its allied techniques (e.g., microbubble contrast agents) for quantifying physiological flow, tissue perfusion, and molecular information, and their applications in cardiovascular diseases and cancer.

Dr. Tang is an Associate Editor of IEEE TRANSACTIONS ON UltrasonICS, FERROELECTRICS, AND FREQUENCY CONTROL, and is on the Editorial Advisory Board of Ultrasound in Medicine and Biology.

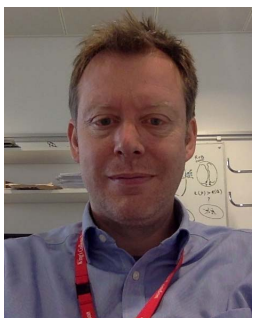

Robert J. Eckersley received the B.Sc. degree in physics from King's College London in 1991 and the Ph.D. degree from the University of London in 1997, while studying with the Institute of Cancer Research, Royal Marsden Hospital, London.

He then went on to work at the Hammersmith Hospital NHS trust and in 1999 was awarded an MRC Research Training fellowship. As part of this fellowship, he spent some time at the University of Toronto at the Sunnybrook Health Sciences Centre. He subsequently continued as a postdoctoral researcher within the Imaging Sciences Department at Imperial College London and became a non-clinical Lecturer in Ultrasound in 2007. In 2012, he joined the Biomedical Engineering Department at King's College London as a Senior Lecturer. His research interests range from fundamental studies to clinical applications. Examples include: Image and signal analysis of ultrasound data for functional imaging or tissue characterisation; nonlinear imaging for improved detection of microbubbles, and understanding errors and artefacts in ultrasound contrast imaging.

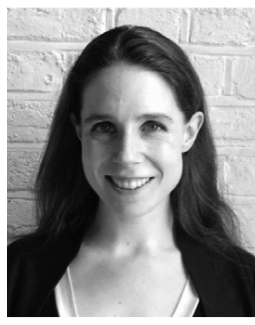

Eleanor Stride received the B.Eng. and Ph.D. degrees in mechanical engineering from University College London, London, U.K.

She was appointed to a lectureship and a Royal Academy of Engineering and Engineering and Physical Sciences Research Council Research Fellowship at the University College London. She joined the Biomedical Ultrasonics, Biotherapy, and Biopharmaceutical Laboratory at the Oxford Institute of Biomedical Engineering, Oxford, U.K., where she became a Professor in 2014. She has authored 125 refereed journal papers, four book chapters, and presented over 100 conference papers. She is involved in the development of new patented technologies for the preparation of microbubble suspensions for ultrasound imaging and drug delivery, and she has set up a spin-out company in this area. Her current research interests include the development of systems, which integrate medical imaging and therapy.

Dr. Stride was a recipient of the EPSRC Challenging Engineering grant in 2011. Her work has been recognized through the award of a Philip Leverhulme Prize, the EPSRC and the Journal of the Royal Society Interface Award, the Engineering Medal at the Parliamentary Science, Engineering, and Technology for Britain awards, the Bruce Lindsay Award from the Acoustical Society of America, and the IET A F Harvey Prize. 